\title{
Investigation of the Migration/Proliferation Dichotomy and its Impact on Avascular Glioma Invasion
}

\author{
K. Böttger ${ }^{1}$, H. Hatzikirou ${ }^{2}$, A. Chauviere ${ }^{2, *}$ and A. Deutsch ${ }^{1}$ \\ ${ }^{1}$ Center for Information Services and High-Performance Computing, Technische Universität \\ Dresden, 01062 Dresden, Germany \\ 2 Department of Pathology, University of New Mexico, Albuquerque, NM 87131, USA
}

\begin{abstract}
Gliomas are highly invasive brain tumors that exhibit high and spatially heterogeneous cell proliferation and motility rates. The interplay of proliferation and migration dynamics plays an important role in the invasion of these malignant tumors. We analyze the regulation of proliferation and migration processes with a lattice-gas cellular automaton (LGCA). We study and characterize the influence of the migration/proliferation dichotomy (also known as the "Go-or-Grow" mechanism) on avascular glioma invasion, in terms of invasion speed and width of the infiltration zone. We show that the invasive behavior of the (macroscopic) tumor colony is a highly complex phenomenon that cannot be extrapolated by the sole knowledge of the (microscopic) individual cell phenotype.
\end{abstract}

Key words: avascular glioma invasion, cell migration/proliferation, Go-or-Grow, LGCA AMS subject classification: 35Q92, 37B15, 92B05, 92C17

${ }^{*}$ Corresponding author. E-mail: achauviere@salud.unm.edu 


\section{Introduction}

Tumor invasion is one of the major causes of poor clinical outcome in patients suffering from cancer. The development of malignant tumors is characterized by uncontrolled proliferation and dysregulated cell migration. A prominent medical paradigm of highly invasive tumors are malignant gliomas (known as Glioblastoma Multiforme). Gliomas consist of a highly proliferative core mass surrounded by an invasive zone where cells with increased motility (invasive cells) infiltrate adjacent brain tissue [8]. The further this invasive zone expands, the more diffuse the extent of the tumor. Consequently, the surgical removal of the tumor and adjuvant treatments such as radiotherapy and chemotherapy have limited efficacy and cannot generally prevent glioma recurrence. The understanding of mechanisms that govern glioma invasion are thus of high clinical importance.

Giese et al. [9] found that mechanisms regulating cell motility contribute to the development of the invasive phenotype in malignant gliomas. Experimental evidence suggests that there may be an inherent and inverse correlation between cell motility and proliferation within a malignant glioma cell population. In particular, it has been shown that highly migratory glioma cells have a lower proliferation rate compared to those with weak migratory ability (that proliferate much more). This antagonistic behavior of cell movement and mitotic activity is supported by numerous in vitro and in vivo observations [9] and is referred to as the migration/proliferation dichotomy or "Go-or-Grow" [8]. Recently, Godlweski et al. [10] identified a glioma expressed microRNA that regulates the balance of proliferation and migration in glioma cells in response to metabolic stress. Their experimental data reveal that, in addition to inhibiting glioma cell migration, this microRNA expression also promotes cell proliferation. These findings suggest that motility and proliferative events share common signaling pathways, defining a unique intracellular mechanism that regulates both phenomena [10]. Therefore, a better understanding of the regulation of tumor cell migration and proliferation can provide new insights into the transition from a proliferative to an invasive tumor phenotype, thus allowing for the development of innovative therapeutic strategies.

The influence of the "Go-or-Grow" mechanism on glioma invasion has been recently the subject of several mathematical investigations. The work by Athale et al. [1] is based on a molecular interaction network of genes and proteins to simulate the dichotomy between cell migration and proliferation. The model shows that the behavioral decisions made at the single cell level impact the spatial dynamics of the entire cancerous lesion. Mansury and colleagues [16] introduced evolutionary game theory into an agent-based brain tumor model. By assuming proliferative and migratory tumor cell sub-populations, the authors show that the dynamics of tumor invasion depends on cell-cell interactions and that there exists an optimal payoff for which the tumor growth is maximized. Another approach was proposed by Fedotov et al. [6], in which the authors study the effect of the "Go-or-Grow" mechanism on the spreading of tumor cells in the invasive zone by means of a two-component reaction-transport model. They find that the resulting dynamics is based on anomalous transport (sub-diffusion), which leads to a decrease of the propagation rate compared to a standard diffusion approximation for transport not accounting for the "Go-or-Grow" mechanism.

More recently, Hatzikirou et al. [12] investigated mechanisms that may promote the progression from benign neoplasms to malignant invasive tumors. Using a lattice-gas cellular automaton, 
the authors model a phenotypically homogeneous, avascular tumor growing in a homogeneous oxygen concentration field and identify the fittest tumor phenotype by varying the oxygen stress. They conclude that the transition to an invasive tumor phenotype can be explained on the basis of the intracellular "Go-or-Grow" mechanism regulated by oxygen shortage in the environment of a growing tumor. The study performed by Pham et al. [17] is based on a reaction-diffusion framework to study the dynamics of a population of glioma cells switching from a migratory to a proliferative phenotype (and vice-versa) due to the local cell density. Their model shows that the density-dependent "Go-or-Grow" mechanism can produce complex dynamics associated with tumor heterogeneity during invasion. In another recent study, Tektonidis et al. [22] compared simulation results from a lattice-gas cellular automaton model to in vitro glioma invasion data. They identify the migration/proliferation dichotomy as a central mechanism in glioma invasion to explain the experimental features of the glioma's infiltrative zone.

In this paper, we are interested in quantifying the influence of the "Go-or-Grow" mechanism on avascular glioma invasion. We present a model that incorporates specific tumor cell dynamics and analyze in detail the interplay of proliferation and migration processes. We investigate the spatiotemporal dynamics of two cell populations with different migratory and proliferative abilities and model the "Go-or-Grow" mechanism as cells switching between mutually exclusive migratory and proliferative phenotypes, as proposed by the authors in [22]. The central aspect of our study is the characterization and the quantification of the invasive tumor front dynamics. To the best of our knowledge, none of the existing studies have thoroughly analyzed the influence of the "Go-orGrow" mechanism on the invasive front of the tumor. Here, we focus on the following questions:

- How does the "Go-or-Grow" mechanism influence the width of the invasive zone?

- How does the "Go-or-Grow" mechanism influence the expansion speed of the invasive tumor front?

In the following section, we propose a lattice-gas cellular automaton (LGCA) [5] model for the "Go-or-Grow" mechanism. Our model assumes that the tumor colony is split into two interacting sub-populations that represent the motile and proliferative phenotypes, with tumor cells switching between these phenotypes. In Section 3, we investigate the influence of the "Go-or-Grow" mechanism on tumor invasion and perform a parameter study by means of numerical simulations. In Section 4, we derive various continuum macroscopic approximations of the LGCA model and discuss their ability to capture features observed in the LGCA simulations and associated with the "Go-or-Grow" mechanism. Finally, we discuss our results and their clinical implications, and propose directions for future investigations.

\section{Lattice-gas cellular automaton model}

Lattice-gas cellular automata allow for a straightforward and intuitive implementation of cell migration and cell kinetics. LGCA models also allow for parallel synchronous movement and fast 
updating of a large number of cells. Both features make LGCA models well-suited for modeling tumor growth and invasion.

We define our "Go-or-Grow" model on a two-dimensional square lattice $\mathcal{L}=L_{1} \times L_{2} \subseteq \mathbb{Z}^{2}$, where $L_{1}$ and $L_{2}$ are the lattice dimensions. To every lattice node $\mathbf{r} \in \mathcal{L}$ a set of velocity channels $\left(\mathbf{r}, \mathbf{c}_{i}\right), i=1, \ldots, b$ is associated. The parameter $b$ is the coordination number, i.e. the number of velocity channels on a node which coincides with the number of nearest neighbors on a given lattice, that is $b=4$ for a square lattice as that we consider in this paper. Cells move on the discrete lattice with discrete velocities $m \in \mathbb{N}$, i.e. they hop at discrete times $k \in \mathbb{N}$ from a given node to a neighboring one, as determined by the cell velocity. The set of velocities for the square lattice is represented by the two-dimensional channel velocity vectors $\mathbf{c}_{1}=\left(\begin{array}{l}1 \\ 0\end{array}\right), \mathbf{c}_{2}=\left(\begin{array}{l}0 \\ 1\end{array}\right)$, $\mathbf{c}_{3}=\left(\begin{array}{c}-1 \\ 0\end{array}\right), \mathbf{c}_{4}=\left(\begin{array}{c}0 \\ -1\end{array}\right)$ and $\mathbf{c}_{j}=\left(\begin{array}{l}0 \\ 0\end{array}\right), j=b+1, \ldots, \tilde{b}$, where $\tilde{b}=b+\beta$ is the total number of channels per node which can be occupied simultaneously, including $\beta$ arbitrary channels with zero velocity (rest channels). Additionally, LGCA impose an exclusion principle on channel occupation, i.e. each channel may at most host one individual. We represent the healthy tissues by the empty channels and model explicitly two cell types, denoted by $\sigma \in\{r, v\}$ : moving tumor cells $(v)$ and resting tumor cells $(r)$, each population occupying its own lattice $\mathcal{L}_{\sigma}$. The occupation numbers $\eta_{\sigma, i}(\mathbf{r}, k), i=1, \ldots, \tilde{b}, \sigma \in\{r, v\}$ are random Boolean variables that indicate the presence $\left(\eta_{\sigma, i}=1\right)$ or absence $\left(\eta_{\sigma, i}=0\right)$ of one cell in the channel $\left(\mathbf{r}, \mathbf{c}_{i}\right)$. To characterize the state of a node $\mathbf{r} \in \mathcal{L}_{\sigma}$ at time $k \in \mathbb{N}$, we define the node-configuration

$$
\boldsymbol{\eta}_{\sigma}(\mathbf{r}, k):=\left(\eta_{\sigma, 1}(\mathbf{r}, k), \ldots, \eta_{\sigma, \tilde{b}}(\mathbf{r}, k)\right) \in \mathcal{E}
$$

where $\mathcal{E}=\{0,1\}^{\tilde{b}}$ denotes the automaton state space. The total number of cells at a node $\mathbf{r}$ and time $k$ for a given species $\sigma \in\{r, v\}$, also referred to as node density, is defined by

$$
n_{\sigma}(\mathbf{r}, k):=\sum_{i=1}^{\tilde{b}} \eta_{\sigma, i}(\mathbf{r}, k) .
$$

We also define the total node density as the sum of the node densities of both cell types

$$
n(\mathbf{r}, k):=\sum_{\sigma=r, v} \sum_{i=1}^{\tilde{b}} \eta_{\sigma, i}(\mathbf{r}, k)=n_{r}(\mathbf{r}, k)+n_{v}(\mathbf{r}, k) .
$$

\subsection{Dynamics}

The dynamics of our LGCA arise from the repeated application of three operators: propagation $(\mathrm{P})$, reorientation $(\mathrm{O})$ and cell kinetics $(\mathrm{K})$. The propagation and reorientation operators define cell movement, whereas the cell kinetics operator changes the local number of cells on a node (e.g., due to birth processes). The composition $\mathrm{K} \circ \mathrm{O} \circ \mathrm{P}$ of the three operators is applied independently 
at every node $\mathbf{r}$ of the lattice and at each time $k$ to evaluate the configuration at time $k+\tau$, as given by

$$
\eta_{\sigma, i}^{\mathrm{KoO} \circ \mathrm{P}}\left(\mathbf{r}+m_{\sigma} \mathbf{c}_{i}, k+\tau\right)=\mathcal{R}\left(\eta_{\sigma, i}(\mathbf{r}, k)\right),
$$

where $m_{\sigma} \in \mathbb{N}$ determines the speed and $m_{\sigma} \mathbf{c}_{i}$ is the translocation of one cell.

\subsubsection{Cell migration and reorientation}

The cell translocation is modeled by the propagation step. By the application of the propagation operator $(\mathrm{P})$, all cells are transported simultaneously to nodes in the direction of their velocity, i.e. a cell residing in channel $\left(\mathbf{r}, \mathbf{c}_{i}\right)$ at time $k$ is moved to a neighboring channel $\left(\mathbf{r}+m_{\sigma} \mathbf{c}_{i}, \mathbf{c}_{i}\right)$ during one time step $\tau$. Since resting tumor cells do not move, we fix $m_{r}=0$. In terms of occupation numbers, the state of a channel $\left(\mathbf{r}+m_{\sigma} \mathbf{c}_{i}, \mathbf{c}_{i}\right)$ after propagation becomes

$$
\eta_{\sigma, i}^{\mathrm{P}}\left(\mathbf{r}+m_{\sigma} \mathbf{c}_{i}, k+\tau\right)=\eta_{\sigma, i}(\mathbf{r}, k) .
$$

Mass and momentum are conserved during the propagation step.

The reorientation operator $(\mathrm{O})$ is responsible for the redistribution of cells among the velocity channels of a node, providing a new velocity distribution. In this paper, we assume that individual cells perform random walks. The resulting transition probabilities are

$$
P\left(\boldsymbol{\eta}_{\sigma} \rightarrow \boldsymbol{\eta}_{\sigma}^{\mathrm{O}}\right)(\mathbf{r}, k)=\frac{1}{Z} \delta\left(n_{\sigma}(\mathbf{r}, k), n_{\sigma}^{\mathrm{O}}(\mathbf{r}, k)\right),
$$

where the normalization factor $Z=\sum_{\boldsymbol{\eta}_{\sigma}^{\mathrm{O}}(\mathbf{r}, k)} \delta\left(n_{\sigma}(\mathbf{r}, k), n_{\sigma}^{\mathrm{O}}(\mathbf{r}, k)\right)$ corresponds to the equivalence class defined by the value of the pre-interaction node density $n_{\sigma}(r, k)$. Obviously, this case implies a random redistribution of the cells among the node's channels. The Kronecker $\delta$ assumes the mass conservation of this operator. This choice of the reorientation operator is one out of various possible ways to describe random motion by means of a LGCA model, as proposed in [5], and will simplifies the analytical derivation of the equations describing the macroscopic dynamics of the automaton (see Section 4).

\subsubsection{Cell kinetics}

The interactions among individuals of each population are dictated by the "Go-or-Grow" mechanism. We consider the two following processes:

- Phenotypic switch (S): Cells can either rest or move. Phenotypes are changed with constant probabilities $\gamma_{v r}$ and $\gamma_{r v}$ that denote the probabilities of a moving cell to become resting and vice versa. Moving and resting populations can exchange cells if there is enough free space on the corresponding lattice.

- Mitosis (M) (cell doubling process): Resting tumor cells undergo mitosis with probability $r_{m}$. For the creation of a new resting cell on a node, the existence of at least one cell and one free channel are required (i.e., mitosis can occur only if there is enough free space). 
Since, under the "Go-or-Grow" mechanism, moving cells are excluded from having proliferative abilities, the cell kinetics operator for the moving population is reduced to $\mathrm{K}=\mathrm{S}$, while we define $\mathrm{K}=\mathrm{M} \circ \mathrm{S}$ for the resting population.

- Resting tumor cells: Resting tumor cells are allowed to proliferate if there is sufficient space and to change phenotype. The new node density generated through these processes is defined by:

$$
\begin{aligned}
& n_{r}^{\mathrm{S}}(\mathbf{r}, k):= \begin{cases}n_{r}(\mathbf{r}, k)+1 & \text { w.p. } \gamma_{v r} \text { if } n_{v}(\mathbf{r}, k) \geq 1 \wedge n_{r}(\mathbf{r}, k)<\beta \\
n_{r}(\mathbf{r}, k)-1 & \text { w.p. } \gamma_{r v} \text { if } n_{v}(\mathbf{r}, k)<b \wedge n_{r}(\mathbf{r}, k) \geq 1 \\
n_{r}(\mathbf{r}, k) & \text { else, }\end{cases} \\
& n_{r}^{\mathrm{M}}(\mathbf{r}, k):= \begin{cases}n_{r}(\mathbf{r}, k)+1 & \text { w.p. } r_{m} \text { if } n_{r}(\mathbf{r}, k) \geq 1 \wedge n_{r}(\mathbf{r}, k)<\beta \\
n_{r}(\mathbf{r}, k) & \text { else, }\end{cases}
\end{aligned}
$$

where w.p. denotes "with probability".

- Moving tumor cells: Moving tumor cells migrate but are not allowed to proliferate ("Goor-Grow" ) and, like resting cells, can change phenotype. The change of node density due to the phenotypic switch is given by:

$$
n_{v}^{\mathrm{S}}(\mathbf{r}, k):= \begin{cases}n_{v}(\mathbf{r}, k)-1 & \text { w.p. } \gamma_{v r} \text { if } n_{r}(\mathbf{r}, k)<\beta \wedge n_{v}(\mathbf{r}, k) \geq 1 \\ n_{v}(\mathbf{r}, k)+1 & \text { w.p. } \gamma_{r v} \text { if } n_{r}(\mathbf{r}, k) \geq 1 \wedge n_{v}(\mathbf{r}, k)<b \\ n_{v}(\mathbf{r}, k) & \text { else. }\end{cases}
$$

Details of the definition of the post-reaction states $\eta_{\sigma, i}^{K}(\mathbf{r}, k)$ associated with the above definitions can be found in Appendix 6.1.

\subsubsection{Micro-dynamical equations}

The spatio-temporal automaton dynamics are fully specified by the micro-dynamical equations:

$$
\begin{aligned}
\eta_{\sigma, i}^{\mathrm{K}}(\mathbf{r}, k) & =\eta_{\sigma, i}(\mathbf{r}, k)+\mathcal{R}_{\sigma, i}(\mathbf{r}, k), \\
\eta_{\sigma, i}\left(\mathbf{r}+m_{\sigma} \mathbf{c}_{i}, k+\tau\right) & =\sum_{j=1}^{\tilde{b}} \mu_{j}(\mathbf{r}, k) \eta_{\sigma, j}^{\mathrm{K}}(\mathbf{r}, k) .
\end{aligned}
$$

The first equation (2.2) refers to the application of the cell kinetics operator (K). The term $\mathcal{R}_{\sigma, i}$ describes changes in the channel occupation number for resting and moving cells (see details in Appendix 6.1.). The second equation (2.3) refers to the redistribution of cells among the velocity channels and the propagation to the neighboring nodes. The $\mu_{j}(\mathbf{r}, k) \in\{0,1\}$ are Boolean random variables that select only one of the $\tilde{b}$ terms of the right-hand side of equation (2.3). They satisfy 
the relation $\sum_{j=1}^{\tilde{b}} \mu_{j}=1$. As stated in Section 2.1.1., we implement the random walk dynamics as a simple reshuffling of cells, which leads to the probability $1 / \tilde{b}$ of choosing a specific channel.

By combining equations (2.2) and (2.3) the spatio-temporal automaton dynamics is completely described by the micro-dynamical difference equations:

$$
\eta_{\sigma, i}\left(\mathbf{r}+m_{\sigma} \mathbf{c}_{i}, k+\tau\right)-\eta_{\sigma, i}(\mathbf{r}, k)=\eta_{\sigma, i}^{\mathrm{KoO}}(\mathbf{r}, k)-\eta_{\sigma, i}(\mathbf{r}, k) \in\{-1,0,1\}
$$

for $m_{\sigma} \in \mathbb{N}, \sigma \in\{r, v\}$ and $i=1, \ldots, \tilde{b}$.

\subsection{Simulation procedure and analysis}

Our LGCA model is simulated on a two-dimensional square lattice ${ }^{\dagger}$ for 1000 iterations. A typical simulation result obtained by our LGCA is depicted in Figure 1. We fix the number of rest channels $^{\ddagger}$ to $\beta=8$. The initial condition is a small, fully occupied disc in the center of the lattice, i.e. all velocity and rest channels of a node in the disc are occupied.

The core region is identified as a densely populated central area surrounded by a sparsely populated invasive zone. We estimate the core and invasive radii at each iteration of the simulation by assuming that the system is spherically symmetric (which is observed in our numerical simulations). To this end, we define the radial density profile $D(\mathbf{r})$ as the average cell density function of the radial distance from the center of the spheroid

$$
D(\mathbf{r}):=\frac{1}{2 \pi} \int_{\phi=0}^{2 \pi} n(\mathbf{r}, \phi) \mathrm{d} \phi,
$$

where $(\mathbf{r}, \phi)$ are the polar coordinates of the image with origin at the image's center $\left(r_{x 0}, r_{y_{0}}\right)$. Then, we determine the core radius as

$$
R_{\text {core }}=\min _{\mathbf{r}}\left\{\mathbf{r}: D(\mathbf{r})<\theta_{\text {core }}\right\},
$$

where $\theta_{\text {core }} \in(0, \tilde{b})$ is a threshold value.

Following the method in Stein et al. [19] we define a quantity $G$ that represents the local squared density gradient:

$$
\begin{aligned}
G\left(n\left(\mathbf{r}_{i j}\right)\right)=\left(\nabla n\left(\mathbf{r}_{i j}\right)\right)^{2}: & \left(n\left(\mathbf{r}_{i+1, j}\right)-n\left(\mathbf{r}_{i j}\right)\right)^{2}+\left(n\left(\mathbf{r}_{i-1, j}\right)-n\left(\mathbf{r}_{i j}\right)\right)^{2} \\
& +\left(n\left(\mathbf{r}_{i, j+1}\right)-n\left(\mathbf{r}_{i j}\right)\right)^{2}+\left(n\left(\mathbf{r}_{i, j-1}\right)-n\left(\mathbf{r}_{i j}\right)\right)^{2},
\end{aligned}
$$

where $\mathbf{r}_{i j}$ is a node coordinate in the lattice. Large values of $G$ indicate rapid spatial fluctuations in the lattice density, which typically occurs in the invasive zone. On the other hand, low values of $G$ are found in the core region and outside the invasive zone. We estimate the average quantity

$$
\bar{G}(\mathbf{r})=\frac{1}{2 \pi} \int_{\phi=0}^{2 \pi} G(n(\mathbf{r}, \phi)) \mathrm{d} \phi,
$$

\footnotetext{
${ }^{\dagger}$ The lattice size is large enough to avoid boundary effects, i.e. the simulated tumor does not reach the lattice boundary.

${ }^{\ddagger}$ Since our model is not applied to a particular experimental validation, the choice of $\beta$ is arbitrary. Qualitatively identical results were obtained for different values of $\beta$.
} 
and define the invasive radius $R_{i n v}$ as the farthest point from the spheroid center where $\bar{G}(\mathbf{r})$ is greater than $\theta_{i n v}$ percent of its maximum:

$$
R_{i n v}=\max _{\mathbf{r}}\left(\mathbf{r}: \bar{G}(\mathbf{r})>\theta_{i n v} \times \max _{\mathbf{r}}(\bar{G}(\mathbf{r}))\right), \theta_{i n v} \in(0,1) .
$$

Both the core $R_{\text {core }}$ and invasive $R_{\text {inv }}$ radii are evaluated at each discrete time of a simulation. Remark that our method for evaluating $R_{\text {core }}$ and $R_{i n v}$ has already been validated in [22] for a particular data set and, for simplicity reason, we maintain the values of $\theta_{\text {core }}$ and $\theta_{i n v}$ used in this previous study.
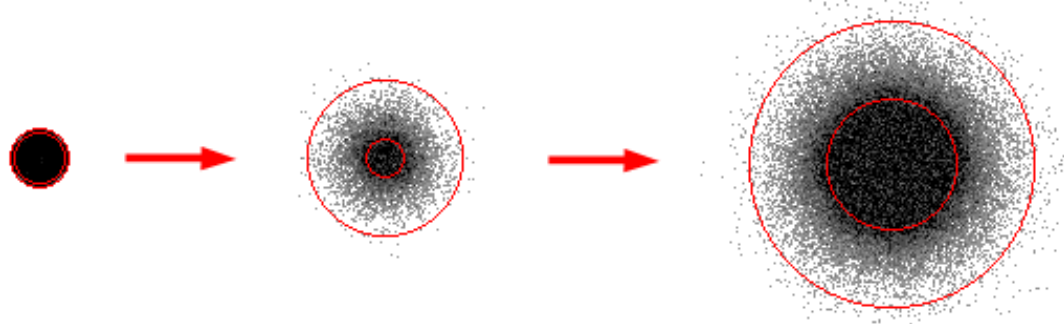

Figure 1: Typical simulation of our LGCA model on a $1000 \times 1000$ lattice starting from an initial fully occupied cluster of nodes in the center of the lattice (radius of 14 nodes). Snapshots at times 0, 250 and 1000 are presented. The inner and outer circles represent the core and invasive radii, respectively, which are evaluated from the definitions (2.6) and (2.9) in the text. The gray scale represents the level of the cell density (light for low values and dark for high values).

Once the two radii are estimated, we derive the following quantities of interest:

1) the width of the invasive zone, defined as the difference between the core and invasive radii

$$
W(t)=R_{\text {inv }}(t)-R_{\text {core }}(t)
$$

Remark that the core and invasive radii are given in lattice unit, which is consequently the unit of the invasive zone width.

2) the expansion speed of the invasive radius, i.e. the time-derivative of $R_{i n v}$. Its evaluation is performed by using a central difference formula. We define

$$
v_{i}=v\left(t_{i}\right)=\frac{R_{i n v}\left(t_{i+1}\right)-R_{i n v}\left(t_{i-1}\right)}{2 \Delta t},
$$

where $t_{i}=t_{0}+i \Delta t$ for $i=1, \ldots, N$. In our study we choose $t_{0}=200, \Delta t=80$ and $N=9$. We neglect the first 200 iterations of the simulation (transient necessary for the creation of the core from the initial fully occupied disc). By doing so, we evaluate the expansion speed corresponding to a well-established growth regime at $N=9$ time points. After the transient, the invasive radius expands linearly. Hence, the values of $N$ and $\Delta t$ are arbitrary and do not affect the results in the case of low speed variations as observed in our simulations. In the following, our main observable 
is the mean expansion speed of the invasive radius, given by

$$
v=\frac{1}{N} \sum_{i=1}^{N} v_{i},
$$

with unit corresponding to lattice unit per time step.

Additionally, our simulations provide the spatio-temporal distributions of the resting and moving populations, and proliferative events (PE), which are used as supplementary material to help understand the dynamics of the whole system.

\section{Numerical investigation}

We study the behavior of the invasive zone as modeled in our LGCA. To this end, we perform a numerical exploration of the space defined by the three model parameters $r_{m}, \gamma_{v r}, \gamma_{r v} \in[0,1]$ and analyze the spatio-temporal evolution resulting from the "Go-or-Grow" mechanism. We use the discretization $r_{m}, \gamma_{v r}, \gamma_{r v} \in\{i \cdot 0.05 ; i=0, \ldots, 20\}$ and investigate the behavior of the invasive zone and its expansion speed for these parameter values.

In order to identify novel behaviors under the influence of the "Go-or-Grow" mechanism, we compare our results with a "control" model. The control model allows for the proliferation of resting and moving cells, i.e. the migration/proliferation dichotomy is not assumed. This model assumes therefore a "Go-and-Grow" behavior, which can generally be described macroscopically by a system of reaction-diffusion equations [2] and, in our specific case, as a Fisher-Kolmogorovlike equation with well-known properties [13].

\subsection{Width of the invasive zone}

We have systematically investigated the features of the invasive zone for the control and the "Goor-Grow" models. Both models lead to similar observations for some parameter ranges, whereas crucial differences occur in specific regions of the parameter space, consistent with preliminary results obtained in [22]. In particular, the interplay between the mitosis probability $r_{m}$ and the motility rate, which depends on the relative strength of $\gamma_{v r}$ and $\gamma_{r v}$, controls the behavior of the invasive zone as explained below.

In the control model, and for almost any combination of the parameter values, the core and invasive radii increase with the same constant expansion speed after a short transient, as illustrated in Figure 2(a) where those radii increase linearly and parallel over time. This gives rise to the formation of an invasive zone of time-invariant width. The sole exception is observed for very low proliferation $\left(r_{m} \ll 1\right)$. In that case, only rare mitotic events occur, while all cells eventually migrate, which may result in the complete loss of the core structure. These two types of behavior are also observed for the "Go-or-Grow" model. When the core structure is maintained, the comparison of the results from the two models, obtained for the same parameter set, underlines the slower dynamics generated by the "Go-or-Grow" mechanism (see Figure 2(a)). Indeed, only a subset of 
the cell population (i.e. the resting cells) can undergo mitosis under the "Go-or-Grow" mechanism, which yields a total number of cells smaller than that of the control case (inlet of Figure 2(a)) and consequently smaller invasive and core radii.

(a)

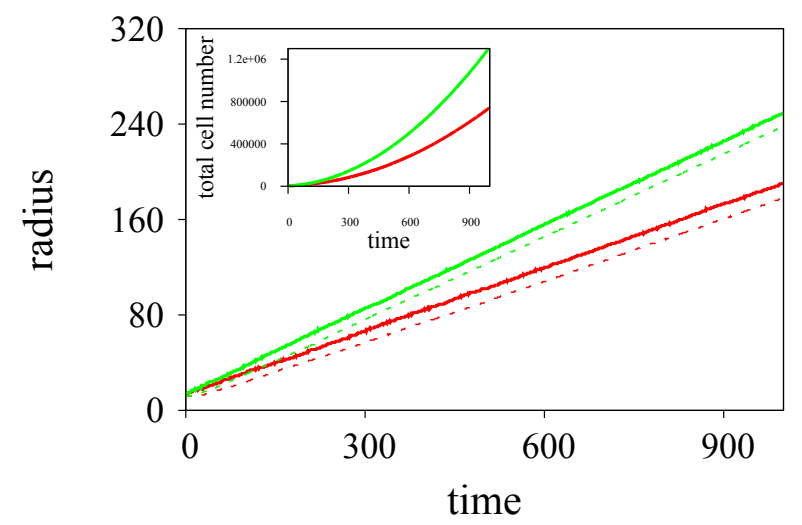

(b)

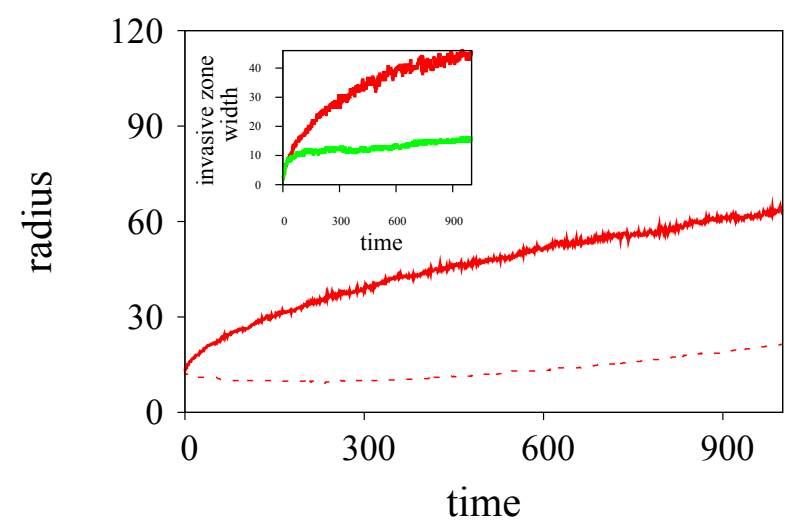

Figure 2: Spatio-temporal evolution of the invasive rim for the control and "Go-or-Grow" models. The figures show the temporal evolution of the core radius (dotted line) and the invasive radius (solid line). Results of the control model are plotted in green against the "Go-or-Grow" model in red. (a) Time-invariant width of the invasive rim. Both models provide the same qualitative behavior with a linear increase of the core and invasive radii over time. The total number of cells differs from one model to the other, as shown in the inlet figure. Parameters are $r_{m}=\gamma_{v r}=\gamma_{r v}=0.2$. (b) Time-increasing width of the invasive rim in the "Go-or-Grow" model. Diverging evolutions of the core and invasive radii are observed. The inlet figure shows the width of the invasive zone for both the control and "Go-or-Grow" models. Parameters are $r_{m}=0.1, \gamma_{v r}=0.01$ and $\gamma_{r v}=0.9$.

A major difference between the two models is observed for sufficiently small mitotic probabilities $r_{m}$ and for probabilities $\gamma_{v r}$ much smaller than $\gamma_{r v}$. For such parameters, the "Go-or-Grow" model generates core and invasive radii with different expansion speeds. This behavior is illustrated in Figure 2(b): the invasive radius grows faster than the core radius, which results in an increasing width of the invasive zone over time (see inlet of the same figure). Note that the difference in speed is actually the result of a slower core expansion due to the following combination of processes: i) since the core is an area of high cell density, proliferation is regulated by space availability; ii) then motile cells escaping from the core and regulated by the "Go-or-Grow" mechanism have low ability to produce new cells in the invasive rim. We shall remark that the divergent nature of the invasive zone width is only a transient. For larger times, the invasive rim and core expansion speeds become eventually identical (results not shown). However, we claim that this transient can be controlled by the parameters. Indeed, we have already observed such a transient in the context of a "Go-or-Rest" dynamics (i.e. in a mass-conservative system where resting cells do not have the ability to proliferate), which is a limit case of the low proliferation characteristic that we consider here. In this unpublished study, we show that a population made of moving and resting cells can undergo anomalous diffusion transients controlled by the total and relative strength of the 
switching rates. Therefore, we suggest that the diverging front behavior may be associated with super-diffusive dynamics generated by cells being more motile and less proliferative.

From the above observations we conclude that the "Go-or-Grow" mechanism, in contrast to the control model, allows for the divergent expansion of the core and invasive radii over time. We have identified that such an atypical behavior occurs for parameter values restricted to a sub-domain of the parameter space characterized by $r_{m} \ll 1$ and $\gamma_{v r} \ll \gamma_{r v}$. This corresponds to a predominantly motile tumor population with low proliferation, which we suggest is the definition of an invasive phenotype that may be associated with super-diffusion transient dynamics. Interestingly, an invasive rim of glioma increasing over time has also been observed in vitro in an invasion assay [20], which suggests that additional experimental investigation of the invasive rim dynamics may be of great interest.

\subsection{Expansion speed of the invasive front}

We define the location of the invasive front of the glioma tumor as the circle of radius $R_{i n v}$ given by equation (2.9). We investigate how fast this front expands away from the tumor bulk and analyze systematically the dependence of the expansion speed of the invasive radius on the model parameters. To this end, we use the discretization of the parameter space proposed in the previous section and fix the value of two of the model parameters $(\in[0,1])$ while varying the value of the third one from 0 to 1 . For our investigation, we exclude however the following peculiar cases. $r_{m}=0$ : core structure not maintained; $\left(\gamma_{v r}=0, \gamma_{r v}=0\right)$ : spreading of the initially moving cells only; $\left(\gamma_{v r}=0, \gamma_{r v}=1\right)$ : purely invasive tumor; $\left(\gamma_{v r}=1, \gamma_{r v}=0\right)$ : no invasion. By doing so, we investigate the effect of microscopic phenotypic changes at the cell level on the invasive ability of the whole tumor cell colony, characterized by the speed of the invasive radius that we denote, from now on, by invasion speed. Our approach allows for the definition of the three cases:

(a) Transition to a more proliferative cell phenotype: by fixing $\left(\gamma_{v r}, \gamma_{r v}\right)$ and increasing $r_{m}$;

(b) Transition to a more resting cell phenotype: by fixing $\left(r_{m}, \gamma_{r v}\right)$ and increasing $\gamma_{v r}$;

(c) Transition to a more motile cell phenotype: by fixing $\left(r_{m}, \gamma_{v r}\right)$ and increasing $\gamma_{r v}$.

Similar to the analysis of the width of the invasive zone, and to provide additional insight into the "Go-or-Grow" mechanism, we compare the invasion speed obtained by the "Go-or-Grow" and the control models. We find that both models can give rise to qualitatively similar and intuitively consistent behaviors during the phenotypic transitions that are defined above. For those cases: transition (a) to a more proliferative phenotype increases the invasion speed; transition (b) to a more resting phenotype decreases the invasion speed; transition (c) to a more motile phenotype increases the invasion speed (see Table 1). These behaviors are consistent with a macroscopic description of the multicellular system, for which the speed of the traveling wave resulting from the growth of the whole tumor colony would be proportional to the product $r^{a} D^{b}(a, b>0)$ where $r$ is the proliferation rate and $D$ the diffusion coefficient [18], the latter being reduced for a more resting phenotype.

However, the "Go-or-Grow" mechanism generates invasive properties at the population (colony) level that are counter-intuitive when considering the phenotypic transitions at the individual (cell) 


\begin{tabular}{||l||c|c||}
\hline \hline $\begin{array}{c}\text { Microscopic - cell level } \\
\text { Phenotypic transition }\end{array}$ & \multicolumn{2}{c|}{$\begin{array}{c}\text { Macroscopic - population level } \\
\text { Invasion speed }\end{array}$} \\
\hline Cases (a), (b) and (c) in the text & Go-and-Grow model & Go-or-Grow model \\
\hline \hline (a) To more proliferative & increase & $\begin{array}{c}\text { increase - Fig. 4(a) } \\
\text { decrease }\end{array}$ \\
\hline (b) To more resting & decrease & $\begin{array}{c}\text { non-monotonic - Fig. 5(a) } \\
\text { increase - Fig. 5(b) } \\
\text { decrease - Fig. 7(b) }\end{array}$ \\
\hline (c) To more motile & increase & non-monotonic - Fig. 7(a) \\
& & increase \\
\hline \hline
\end{tabular}

Table 1: Behavior of the invasion speed associated with the phenotypic transitions for the control ("Go-and-Grow") and "Go-or-Grow" models. Whereas the behavior of the invasion speed is intuitive and always of monotonic nature in the "Go-and-Grow" model (central column), the "Go-or-Grow" model generates behaviors that are counter-intuitive and more complex during the transitions to more resting and more motile phenotype (right column), which is emphasized in bold with referring the reader to the corresponding figures in the text. Each color is associated with a particular behavior of the invasion speed during the phenotypic transitions and is used to define the corresponding region in the parameter space in Figure 3.

(a)

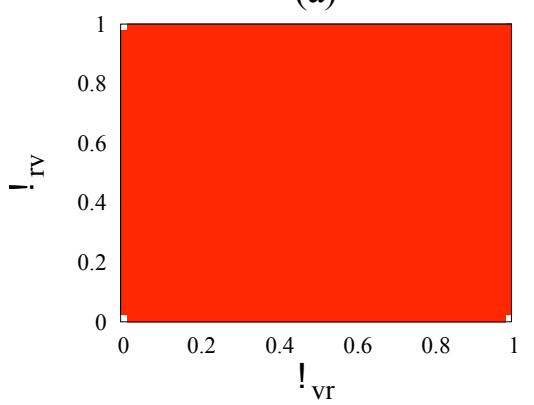

(b)

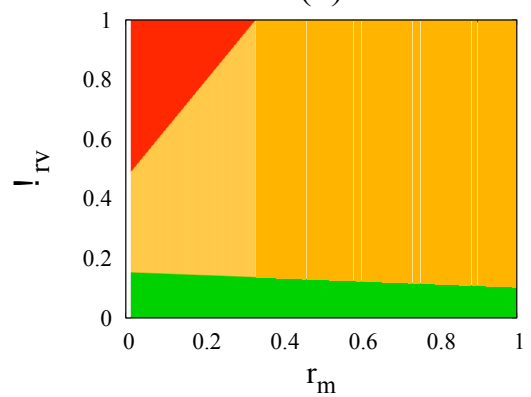

(c)

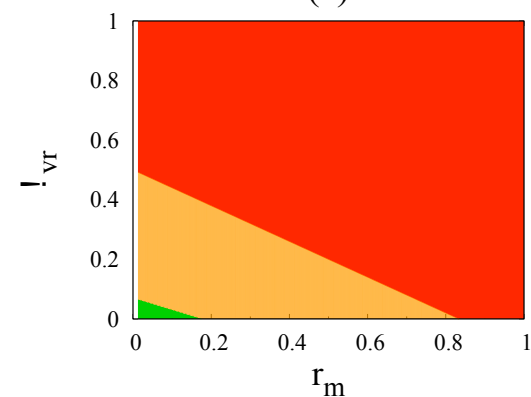

Figure 3: Parameter study of the influence of the phenotypic transitions on the invasion speed for the "Go-or-Grow" model. Each figure shows the behavior of the invasion speed during a phenotypic transition: (a) toward a more proliferative phenotype, i.e. by increasing $r_{m}$ at each point of the plane $\left(\gamma_{v r}, \gamma_{r v}\right)$; (b) toward a more resting phenotype, i.e. by increasing $\gamma_{v r}$ at each point of the plane $\left(r_{m}, \gamma_{r v}\right)$; (c) toward a more motile phenotype, i.e. by increasing $\gamma_{r v}$ at each point of the plane $\left(r_{m}, \gamma_{v r}\right)$. The colors correspond to the three different behaviors: increasing (red), non-monotonic (orange) and decreasing (green), as defined in Table 1.

level. In particular, we find for the "Go-or-Grow" model that the behavior of the invasion speed during the transitions to more resting (b) and more motile (c) phenotypes can also be either nonmonotonic or of opposite monotonic nature than that of the "Go-and-Grow" model (see Table 1). 
The two models are based on the interplay between migration and proliferation, where migration processes are affected by resting phases. There is however a major difference that explains the richness of the behaviors generated by the "Go-or-Grow" model. Indeed, in the latter, the presence of resting phases does not only impact migration features but also the proliferation events, making the invasion speed the result of the competition between two interdependent processes: i) emptying-space, via cell migration, to allow for free space necessary to cell proliferation; ii) filling-space, via proliferation of cells at rest, when free space is available. Therefore, the result of this competition is not trivial and depends on the values of the model parameters, as illustrated in Figure 3.

In the following, and based on the results presented in Figure 3, we have a closer look to each phenotypic transition and propose insight into the dynamics of the invasion speed generated by the "Go-or-Grow" mechanism.

\section{(a) Transition to a more proliferative phenotype}

For an increasing mitotic rate $r_{m}$, each cell becomes potentially more proliferative. For any combination of the exchange parameters (see Figure 3(a)), we observe that the invasion speed increases with $r_{m}$ as illustrated in Figure 4(a). A higher rate of cell duplication leads to an increased frequency of proliferative events, as shown in Figure 4(b). Hence cell proliferation becomes dominant, thus creating more tumor cells that "push" the invasive front even faster.

(a)

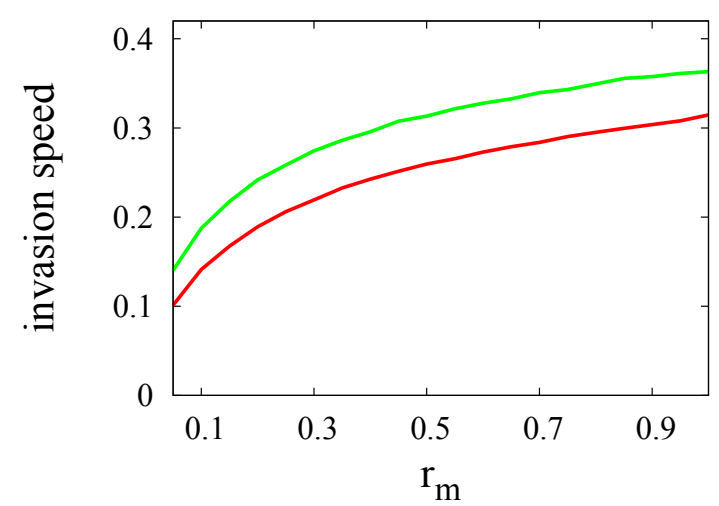

(b)

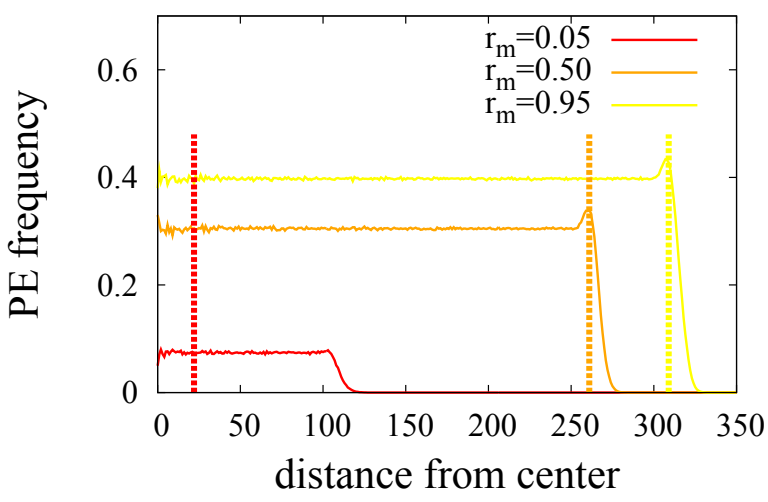

Figure 4: Transition to a more proliferative phenotype (increasing $r_{m}$ for fixed values of $\gamma_{v r}$ and $\gamma_{r v}$ ). (a) Both the control and "Go-or-Grow" models yield an increasing invasion speed. Simulation results (i.e. average over 3 simulations after 1000 iterations, with corresponding standard deviation (STD) less than $10^{-3}$ ) of the "Go-or-Grow" model are plotted in red against the control model in green . Parameters are $\gamma_{v r}=\gamma_{r v}=0.5$. (b) Corresponding spatial distribution (average over 100 simulations) of frequency of proliferative events (PE) in the "Go-or-Grow" model at time 1000 for three different values of $r_{m}$. The vertical dashed lines indicate the location of the core boundary. 


\section{(b) Transition to a more resting phenotype}

Increasing the value of the probability $\gamma_{v r}$ favors a resting phenotype in each cell. Intuitively, one expects that increasing this probability would lead to a decrease of the invasion speed. This behavior is indeed observed for low values of $\gamma_{r v}$ (green region in Figure 3(b)) for which the process of filling-space (due to cells stopping) becomes locally faster than emptying-space (due to cells moving) during the phenotypic transition. However, there are parameter ranges $\left(r_{m}, \gamma_{r v}\right)$ for which we observe non-intuitive behaviors of the invasion speed.

Non-monotonic invasion speed - Figure 5(a). This behavior is observed for combinations of $\left(r_{m}, \gamma_{r v}\right)$ in the orange region in Figure 3(b). For very low values of $\gamma_{v r}$ cell proliferation remains low within the core because tumor cells are not resting enough (see Figure 6(a)), which leads to comparatively low invasion speeds. Subsequently, as $\gamma_{v r}$ increases, tumor cells rest more, which allows proliferation to be enhanced and support a faster invasion. However, the competition of emptying- and filling-space processes introduces an optimal value of $\gamma_{v r}$ that generates a maximum invasion speed. A further increase of $\gamma_{v r}$ beyond this critical value leads to a decreasing invasion speed, which is the intuitively expected behavior since larger values of $\gamma_{v r}$ reduce cell motility, thus also reducing space availability for new proliferation events, as shown in Figure 6(a) where the core radius decreases.

(a)

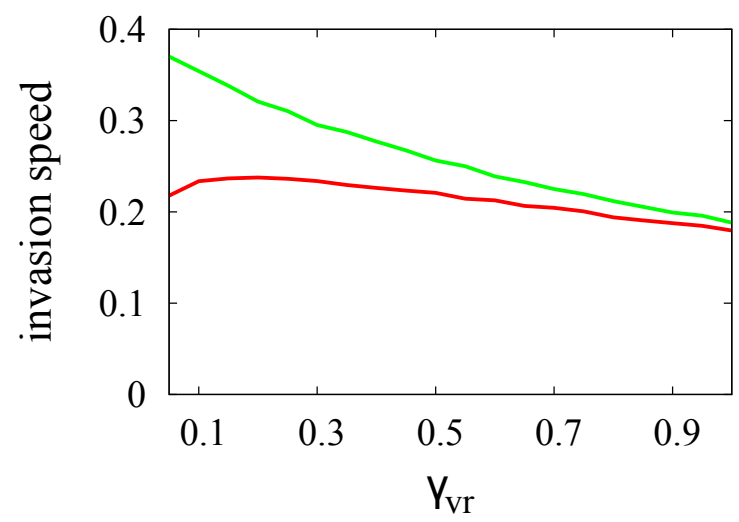

(b)

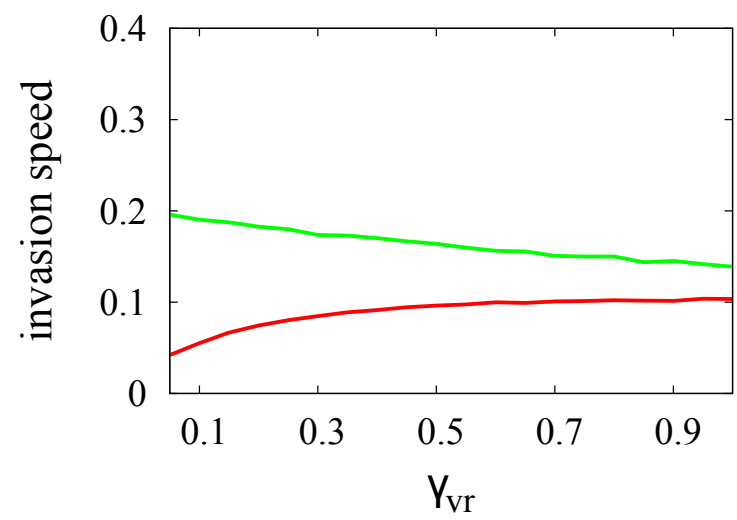

Figure 5: Invasion speed for the control and "Go-or-Grow" models during the transition to a more resting phenotype (increasing $\gamma_{v r}$ for fixed values of $r_{m}$ and $\gamma_{r v}$ ). Simulation results (i.e. average over 3 simulations after 1000 iterations, with corresponding STD $\leq 10^{-3}$ ) of the "Goor-Grow" model are plotted in red against the control model in green. In both figures the control model shows a decreasing invasion speed. (a) Non-monotonic invasion speed in the "Go-or-Grow" model for $r_{m}=0.5$ and $\gamma_{r v}=0.25$. (b) Increasing invasion speed in the "Go-or-Grow" model for $r_{m}=0.05$ and $\gamma_{r v}=0.95$.

Increasing invasion speed - Figure 5(b). This behavior is observed for combinations of $\left(r_{m}, \gamma_{r v}\right)$ in the red region in Figure 3(b). Counterintuitively, the tumor colony expands faster as $\gamma_{v r}$ increases, i.e. for more resting phenotypes. Due to both low values of $r_{m}$ and sufficiently high values of $\gamma_{r v}$, cells have poor proliferative abilities and tend to remain mainly in the motile phenotype. For increasing values of $\gamma_{v r}$ the proliferative events increase in frequency and span further away 
from the core center as illustrated on Figure 6(b). This results from the high rates of $\gamma_{r v}$ which turn the cells into motile and promote invasion simultaneously by moving away from the colony center and by freeing space that allows for additional PEs. Compared to Figure 6(a) the PE frequency has smaller values and its spatial distribution is not restricted to the region of the tumor core.

(a)

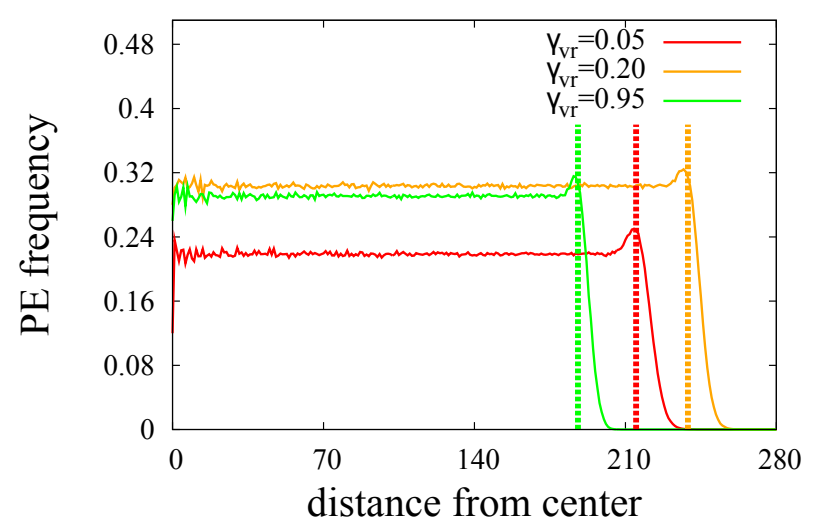

(b)

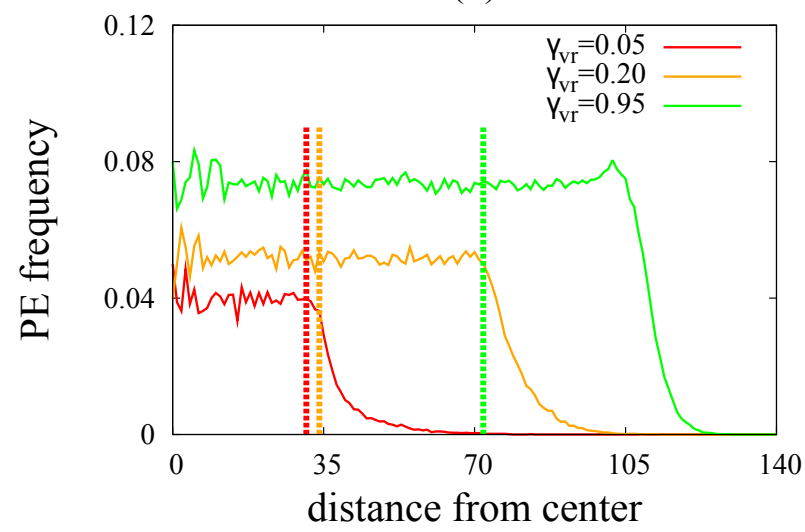

Figure 6: Frequency of proliferative events (PE) in the "Go-or-Grow" model during the transition to a more resting phenotype (increasing $\gamma_{v r}$ for fixed values of $r_{m}$ and $\gamma_{r v}$ ). The figures show the average number (over 100 simulations) of PE per lattice node at time 1000 as a function of the distance from the colony center, and correspond to the invasion speeds in Figures 5(a) and (b), respectively. The vertical dashed lines indicate the location of the core boundary. Parameters are $r_{m}=0.5, \gamma_{r v}=0.25$ (a) and $r_{m}=0.05, \gamma_{r v}=0.95$ (b).

\section{(c) Transition to a more motile phenotype}

Increasing the value of the probability $\gamma_{r v}$ favors a motile phenotype in each cell. Intuitively, one would expect that the invasion speed would consequently increase during the transition. This is indeed observed in the simulations for the parameters $r_{m}$ and $\gamma_{v r}$ in the red region in Figure 3(c). For other combinations of these parameters, we observe the following:

Non-monotonic invasion speed - Figure 7(a). This behavior is observed for combinations of $\left(r_{m}, \gamma_{v r}\right)$ in the orange region in Figure 3(c). Sufficiently low values of $r_{m}$ and $\gamma_{v r}$ (but still larger than $\gamma_{r v}$ to support the difference between motile and resting populations shown in Figure 9(a)) lead to a reduced effective proliferation rate and, consequently, low invasion speeds. By increasing $\gamma_{r v}$ cell motility increases, which, in turn, frees more space to allow for more proliferation, eventually resulting into higher invasion speeds. The competition between emptying- and fillingprocesses drives the system to an optimal invasive behavior. Further increase of $\gamma_{r v}$ leads to a significant reduction of the PE in the core, as shown in Figure 8(a), and eventually to a decrease of the invasion speed of the whole tumor colony.

Decreasing invasion speed - Figure 7(b). This behavior is observed for combinations of $\left(r_{m}, \gamma_{v r}\right)$ in the green region in Figure 3(c). The combination of very low values of $r_{m}$ and $\gamma_{v r}$ 
forces a predominantly migrating cell colony, as supported by Figure 8(b) showing that the frequency of PE is very low (compared to Figures 6(a),(b) and 8(a)). The higher the value of $\gamma_{r v}$, the more time cells spend migrating rather than resting and proliferating. This induces the quasielimination of proliferation, making the population effectively diffusive, and consequently imposes a strong limitation on the invasion speed that is not supported by the core growth.

(a)

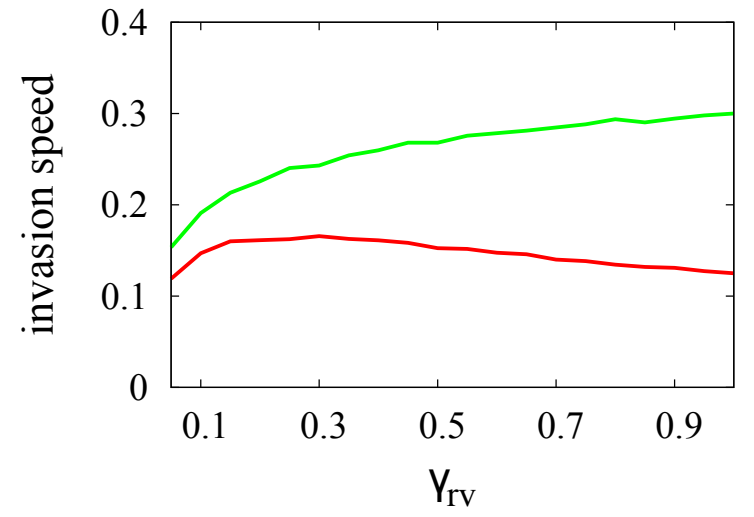

(b)

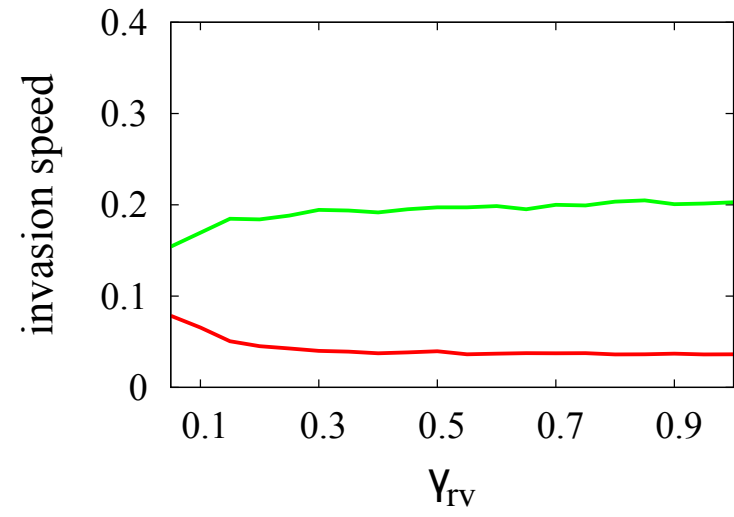

Figure 7: Invasion speed for the control and "Go-or-Grow" models during the transition to a more motile phenotype (increasing $\gamma_{r v}$ for fixed values of $r_{m}$ and $\gamma_{v r}$ ). The figures show an average over 3 simulations after 1000 iterations (corresponding STD $\leq 10^{-3}$ ). Simulation results of the "Go-or-Grow" model are plotted in red against the control model in green. In both figures the control model shows an increasing invasion speed. (a) Non-monotonic invasion speed in the "Go-or-Grow" model for $r_{m}=0.15$ and $\gamma_{v r}=0.15$. (b) Decreasing invasion speed in the "Go-orGrow" model for $r_{m}=0.05$ and $\gamma_{v r}=0.01$.

(a)

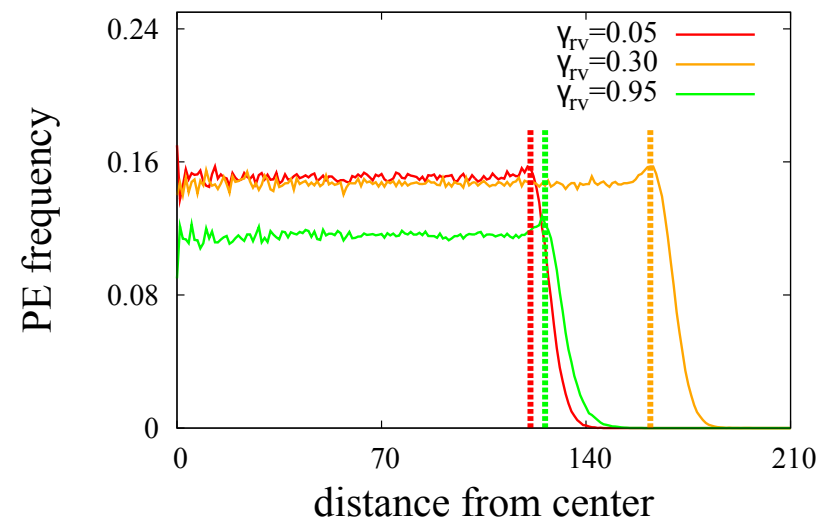

(b)

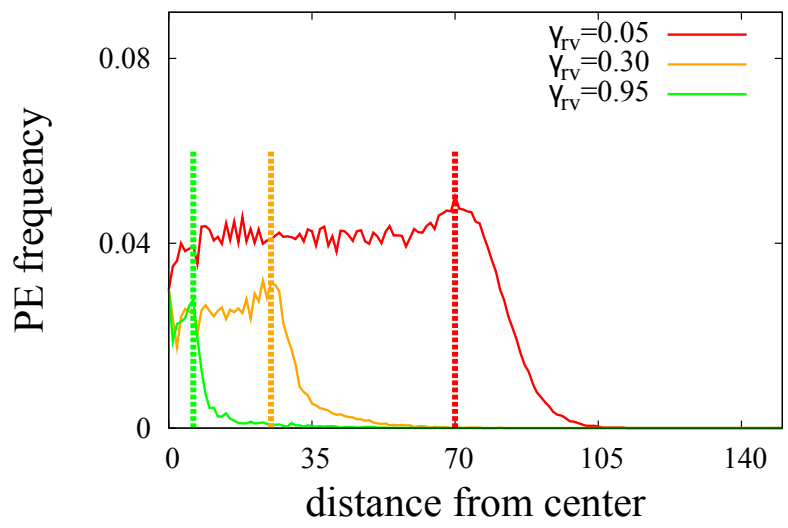

Figure 8: Frequency of proliferative events (PE) in the "Go-or-Grow" model during the transition to a more motile phenotype (increasing $\gamma_{r v}$ for fixed values of $r_{m}$ and $\gamma_{v r}$ ). The figures show the average number (over 100 simulations) of PE per lattice node at time 1000 as a function of the distance from the colony center, and correspond to the invasion speeds in Figures 7(a) and (b), respectively. The vertical dashed lines indicate the location of the core boundary. Parameters are $r_{m}=0.15, \gamma_{v r}=0.15$ (a) and $r_{m}=0.05, \gamma_{v r}=0.01$ (b). 
(a)

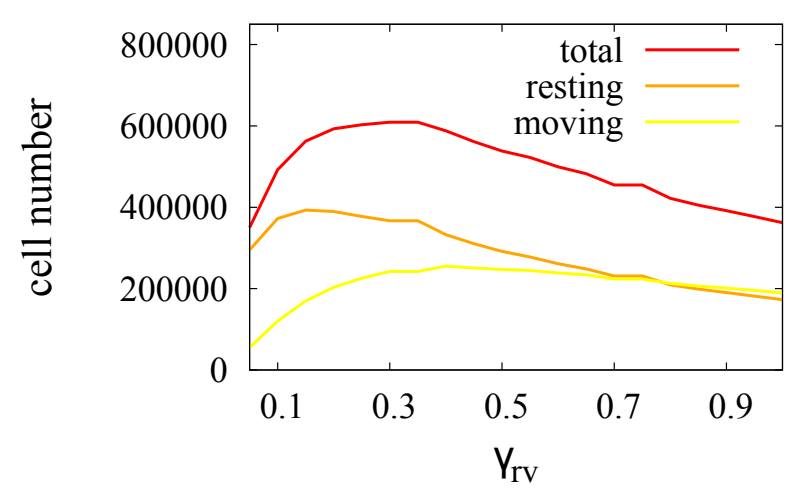

(b)

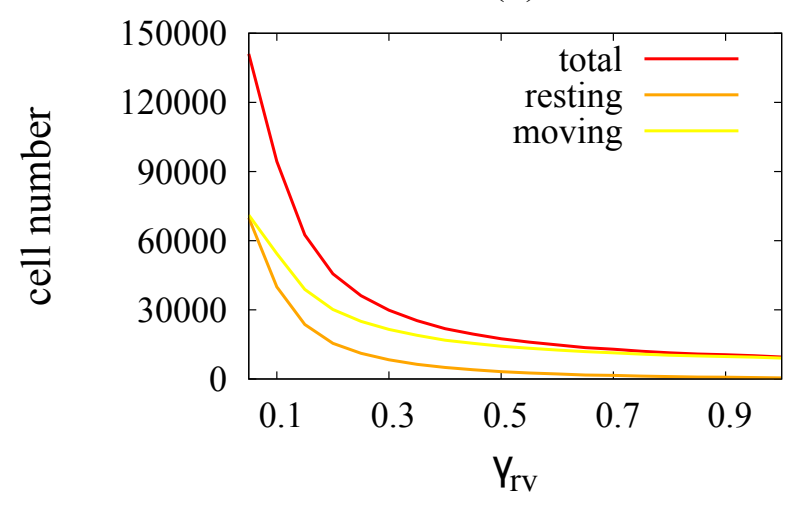

Figure 9: Total number of moving and resting cells in the "Go-or-Grow" model during the transition to a more motile phenotype (increasing $\gamma_{r v}$ for fixed values of $r_{m}$ and $\gamma_{v r}$ ). The figures show an average over 100 simulations at time 1000. The figures correspond to the invasion speeds presented in Figures 7(a) and (b), respectively, for parameters $r_{m}=0.15, \gamma_{v r}=0.15$ (a) and $r_{m}=0.05, \gamma_{v r}=0.01(\mathrm{~b})$.

\subsection{Conclusion}

We have numerically investigated the behavior of the width of the invasive zone and the invasion speed for both the "Go-and-Grow" and the "Go-or-Grow" models. In our parameter study, we have compared these observables in both cases, which allows us to state the two following major features resulting from the "Go-or-Grow" mechanism:

1) A rather motile population of tumor cells $\left(\gamma_{v r} \ll \gamma_{r v}\right)$ with low proliferation $\left(r_{m} \ll 1\right)$ regulated by the "Go-or-Grow" mechanism can generate a time-divergent invasion rim around the tumor core. In such a configuration, the corresponding speed of invasion of the colony remains slow although the phenotype at the individual cell level is mostly motile.

2) Counter-intuitively, a population of tumor cells with high proliferative potential (high value of $r_{m}$ and $\gamma_{v r}$ ), regulated by the "Go-or-Grow" mechanism, has the ability to give rise to a lower number of offspring cells than a more motile population does. This intriguing behavior is emphasized in Figure 6(a) and is consistent with a previous study [12] (although the latter is based on a simpler modeling approach of the "Go-or-Grow" mechanism, which supports the consistency of our results).

\section{Mathematical analysis of the model}

We aim to provide a more complete understanding of the behavior of our LGCA model and propose to derive corresponding mean-field approximations that can be mathematically investigated. The main idea behind such an approach is to replace the description of interactions among many cells by a simplified description based on average (or effective) interactions. This reduces the multicellular 
nature of the problem into an effective formulation that can be stated in the form of macroscopic deterministic descriptions such as ordinary (ODE) or partial (PDE) differential equations.

We propose to derive consecutively the macroscopic mathematical formulations of the nonspatial and spatially-distributed dynamics of our LGCA: the first formulation corresponds to a well-stirred system; the second one is based on the Chapman-Enskog expansion method [4] and yields a reaction-diffusion equations (RDE) system. We analyze and investigate the validity of these mean-field approximations via direct comparison to the LGCA dynamics.

\subsection{Stochastic analysis: Markov chain dynamics}

The stochastic dynamics of our system (Section 2.1.3.) satisfy the Markov assumption, i.e. the system is memoryless. The time evolution of resting and moving tumor cells on a single node $\mathbf{r}$ of the lattice can be described by a Markov chain $\left\{\left(n_{r}, n_{v}\right)_{k}\right\}_{k \in \mathbb{N}}$. The state space is given by $S=\left\{\left(n_{r}, n_{r}\right) \mid n_{r} \in\{0, \ldots, \beta\}, n_{v} \in\{0, \ldots, b\}\right\}$, i.e. there are $|S|=(\beta+1)(b+1)$ possible states. Let $\mathbf{W}$ be the transition matrix of the Markov chain, where

$$
\mathbf{W}\left[\left(n_{r}, n_{v}\right),\left(n_{r}^{\prime}, n_{v}^{\prime}\right)\right]=P\left[\left(n_{r}^{\prime}, n_{v}^{\prime}\right) \mid\left(n_{r}, n_{v}\right)\right]
$$

gives the probability that the Markov chain, starting in state $\left(n_{r}, n_{v}\right)$, ends in state $\left(n_{r}^{\prime}, n_{v}^{\prime}\right)$ after one time step. Due to the fact that we consider the temporal dynamics at a single node, a change in the node density is defined only by the action of the cell kinetics. We present in Figure 10 a tree-diagram that illustrates all possible changes in the node density with state $\left(n_{r}, n_{v}\right)$.

The entries of the transition matrix $\mathbf{W}$ can be derived from the definition of the LGCA cell kinetics. We find that the event probabilities of phenotypic switch, i.e. from the moving to resting phenotype $\left(S_{v r}\right)$ and from the resting to moving phenotype $\left(S_{r v}\right)$, and mitosis $(M)$ are given by:

$$
\begin{aligned}
& P\left(\left\{\mathrm{~S}_{v r}\right\}\right)=\gamma_{v r} \frac{n_{v}}{b}\left(\frac{\beta-n_{r}}{\beta}\right), \\
& P\left(\left\{\mathrm{~S}_{r v}\right\}\right)=\gamma_{r v} \frac{n_{r}}{\beta}\left(\frac{b-n_{v}}{b}\right), \\
& P(\{\mathrm{M}\})=r_{m} \frac{n_{r}}{\beta}\left(\frac{\beta-n_{r}}{\beta}\right) .
\end{aligned}
$$

The event probabilities of mitosis after a switching event $S_{v r}$ or $S_{r v}$ are denoted by $P_{v r}(\{M\})$ and $P_{r v}(\{M\})$, respectively. They are evaluated from a combination of equations (4.1)-(4.3), which yields:

$$
\begin{aligned}
& P_{v r}(\{\mathrm{M}\})=r_{m}\left(\frac{n_{r}+1}{\beta}\right)\left(\frac{\beta-n_{r}-1}{\beta-1}\right), \\
& P_{r v}(\{\mathrm{M}\})=r_{m}\left(\frac{n_{r}-1}{\beta-1}\right)\left(\frac{\beta-n_{r}-1}{\beta}\right) .
\end{aligned}
$$




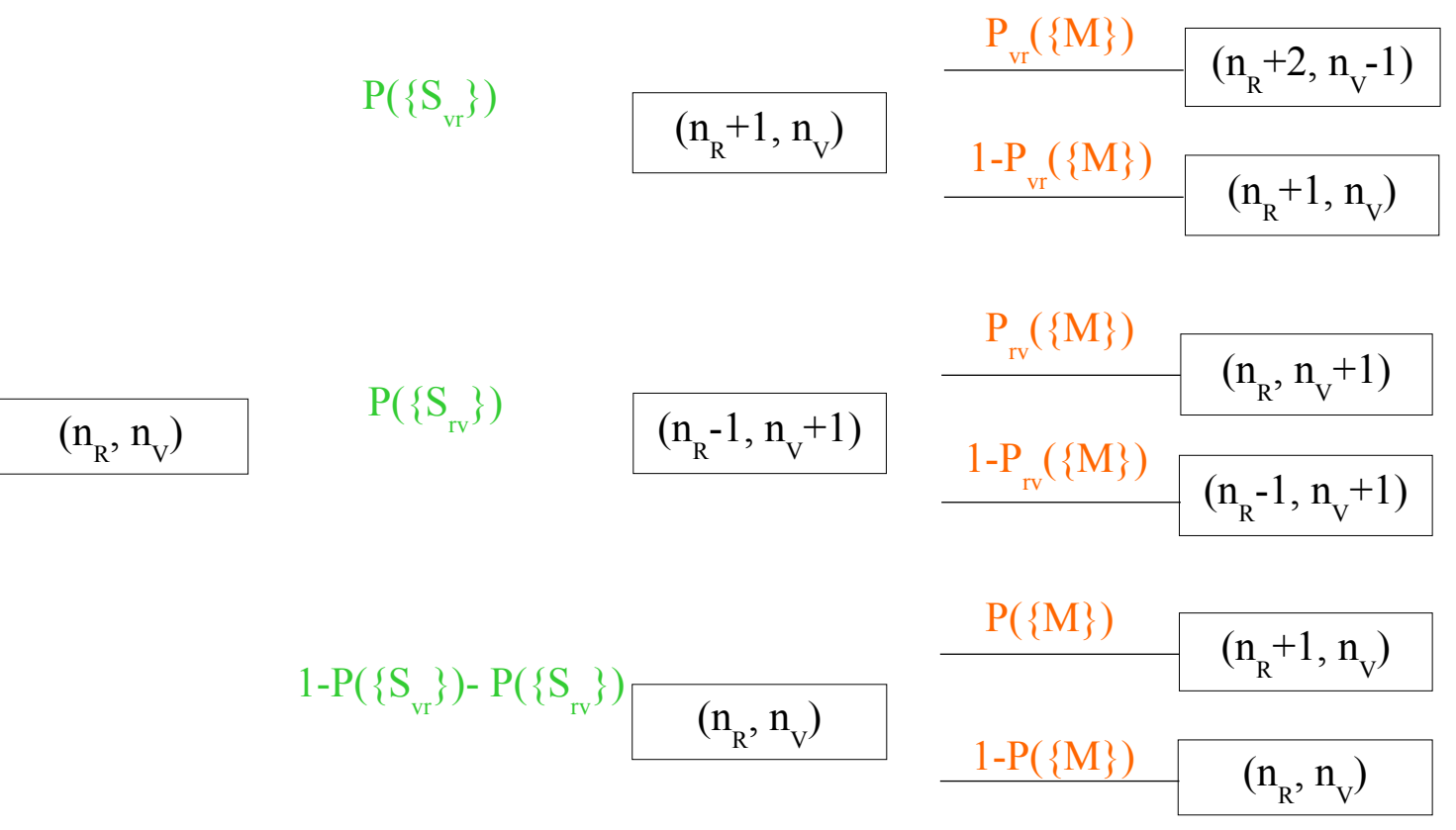

\section{PHENOTYPIC} SWITCH

\section{MITOSIS}

Figure 10: Tree-diagram for the action of the LGCA cell kinetics (phenotypic switch and mitosis). $P\left(\left\{\mathrm{~S}_{v r}\right\}\right), P\left(\left\{\mathrm{~S}_{r v}\right\}\right)$ and $P(\{\mathrm{M}\})$ denote the probabilities of a phenotypic switch from the moving to resting phenotype $\left(S_{v r}\right)$, from the resting to moving phenotype $\left(S_{r v}\right)$, and mitosis $(M)$, respectively. The probabilities of mitotic event after a switching event $S_{v r}$ or $S_{r v}$ are denoted by $P_{v r}(\{M\})$ and $P_{r v}(\{M\})$, respectively. All event probabilities are derived from the LGCA formulation (see text).

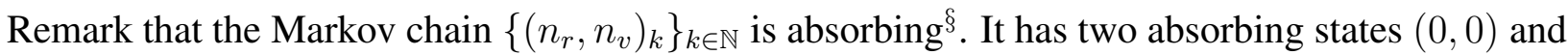
$(\beta, b)$, i.e. defined by $\mathbf{W}[(0,0),(0,0)]=\mathbf{W}[(\beta, b),(\beta, b)]=1$. The probability of absorbing into state $(0,0)$ is zero, since death of cells is not considered in the model .

\subsection{Non-spatial mean-field analysis}

\subsubsection{Well-stirred dynamics}

Assuming a well-stirred system, the system is homogenized and relaxes to a single binomial distribution over the lattice. We apply the mean-field approximation, i.e. we neglect spatial correlations for a given time step of the LGCA. This assumption facilitates the calculation of the node density distribution of individuals over the lattice, which coincides with a spatially homogeneous node density distribution. This allows for the calculation of the deterministic net changes occurring in

\footnotetext{
${ }^{\S} \mathrm{A}$ Markov chain is absorbing if it has at least one absorbing state and if it is possible to get from each nonabsorbing state to some absorbing state in one or more time steps.
} 
the node density between two consecutive times $k$ and $k+\tau$ :

$$
\rho_{\sigma}(k+\tau)-\rho_{\sigma}(k)=F_{\sigma}\left(\rho_{r}, \rho_{v}\right), \sigma \in\{r, v\} .
$$

We refer the reader to Appendix 6.2. for the mathematical derivation of the time-dependent meanfield equation (4.6), in which $F_{r}$ and $F_{v}$ are the mean-field growth laws for the resting and moving cells, respectively. We find that

$$
\begin{aligned}
F_{r}\left(\rho_{r}, \rho_{v}\right) & =r_{m} \frac{\rho_{r}\left(\beta-\rho_{r}\right)}{\beta^{2}}+\gamma_{v r} \frac{\rho_{v}\left(\beta-\rho_{r}\right)}{b \beta}-\gamma_{r v} \frac{\rho_{r}\left(b-\rho_{v}\right)}{b \beta} \\
& +r_{m} \gamma_{v r} \frac{\rho_{v}\left(\beta-\rho_{r}\right)\left(\beta^{2}-\beta-\beta \rho_{r}-\rho_{r}^{2}\right)}{\beta^{3}(\beta-1) b} \\
& +r_{m} \gamma_{r v} \frac{\rho_{r}\left(b-\rho_{v}\right)\left(\beta^{2}+\beta-3 \beta \rho_{r}+\rho_{r}^{2}\right)}{\beta^{3}(\beta-1) b} \\
F_{v}\left(\rho_{r}, \rho_{v}\right) & =\gamma_{r v} \frac{\rho_{r}\left(b-\rho_{v}\right)}{b \beta}-\gamma_{v r} \frac{\rho_{v}\left(\beta-\rho_{r}\right)}{b \beta} .
\end{aligned}
$$

The transition from microscopic processes to a macroscopic description requires a temporal scaling, i.e. a relation between the macroscopic and microscopic variables. We assume the existence of a small parameter $\epsilon \propto \tilde{b}^{-1} \ll 1$ that scales the time variable $t$ :

$$
t=\epsilon k \text {. }
$$

We use the Taylor expansion $\left.\rho_{\sigma}(t)\right|_{t=k+\tau}=\left.\rho_{\sigma}(t)\right|_{t=k}+\left.\epsilon \tau \frac{\mathrm{d} \rho_{\sigma}(t)}{\mathrm{d} t}\right|_{t=k}$ to rewrite (4.6) in continuous variables and obtain a system of two first-order nonlinear ODEs that generically write

$$
\frac{\mathrm{d} \rho_{\sigma}}{\mathrm{d} t}=\frac{1}{\epsilon \tau} F_{\sigma}\left(\rho_{r}, \rho_{v}\right), \sigma \in\{r, v\} .
$$

Due to the structure of the growth laws (4.7) and (4.8) we conclude that the parameters $r_{m}, \gamma_{r v}$ and $\gamma_{v r}$ should be proportional to the scaling parameter $\epsilon$. Therefore, the macroscopic growth laws, i.e. the terms in $\mathcal{O}(\epsilon)$ in $F_{r}$ and $F_{v}$, reduce to

$$
\begin{aligned}
& \bar{F}_{r}\left(\rho_{r}, \rho_{v}\right)=\frac{r_{m}}{\beta} \rho_{r}\left(1-\frac{\rho_{r}}{\beta}\right)-\bar{F}_{v}\left(\rho_{r}, \rho_{v}\right), \\
& \bar{F}_{v}\left(\rho_{r}, \rho_{v}\right)=\frac{\gamma_{r v}}{\beta} \rho_{r}\left(1-\frac{\rho_{v}}{b}\right)-\frac{\gamma_{v r}}{b} \rho_{v}\left(1-\frac{\rho_{r}}{\beta}\right) .
\end{aligned}
$$

Remark that the proliferation of the resting population appears in (4.10) in the form of a logistic growth. This finalizes the derivation of the mean-field approximation of our LGCA model, treated as a well-stirred system, which writes

$$
\left\{\begin{aligned}
\frac{\mathrm{d} \rho_{r}}{\mathrm{~d} t} & =\bar{F}_{r}\left(\rho_{r}, \rho_{v}\right), \\
\frac{\mathrm{d} \rho_{v}}{\mathrm{~d} t} & =\bar{F}_{v}\left(\rho_{r}, \rho_{v}\right),
\end{aligned}\right.
$$

where $\bar{F}_{r}$ and $\bar{F}_{v}$ are given by (4.10) and (4.11), respectively. 


\subsubsection{Stability of the steady-states}

We investigate the non-spatial behavior of our model, i.e. the kinetics resulting from the "Go-orGrow" mechanism, and compare the results of the stochastic and deterministic approaches. We start by recalling the asymptotic properties of the underlying Markov process found in Section 4.1. By studying the temporal evolution process $\left\{\left(n_{r}, n_{v}\right)_{k}\right\}_{k \in \mathbb{N}}$, the states $(0,0)$ and $(\beta, b)$ are found to be absorbing, i.e. any initial state $\left(n_{r}, n_{v}\right)$ will end up into one of those two absorbing states.

The deterministic system (4.12), i.e. the non-spatial mean-field approximation of our LGCA model, admits two steady-states satisfying $\bar{F}_{r}=0$ and $\bar{F}_{v}=0$. From expressions (4.10) and (4.11) we find that these steady-states are $\left(\bar{\rho}_{r}, \bar{\rho}_{v}\right)=(0,0)$ and $(\beta, b)$. To determine the local stability of each steady-state we consider the small perturbation

$$
\left(\delta \rho_{r}, \delta \rho_{v}\right)=\left(\rho_{r}-\bar{\rho}_{r}, \rho_{v}-\bar{\rho}_{v}\right),
$$

to further study the eigenvalues of the Jacobian matrix obtained from the linearized system of equations governing the evolution of this perturbation. In the case $r_{m}=\gamma_{v r}=\gamma_{r v}=0$ the stability of the steady-state solutions cannot be determined; therefore, we assume that at least one of the model parameters is different from zero. After some classic algebra, we find that the steady-state $(0,0)$ is a saddle point, while $(\beta, b)$ is asymptotically stable. We remark that, whereas the steady-state $(0,0)$ is unstable in the deterministic system (4.12), its equivalent is stable in the underlying stochastic description. This is known as the Keizer's paradox [7] where the stochastic system approaches the point $(0,0)$ following a timescale that increases with the system's volume, which makes this state practically inaccessible for very large systems, i.e. in a regime at which the deterministic equation is valid.

In both stochastic and deterministic representations, the fixed-point $(0,0)$ corresponds to the absence of tumor cells (moving and resting) and consequently represents the healthy tissue. The tumor region (where no healthy tissue remains) is associated with the second fixed-point $(\beta, b)$. Therefore, the stability features of the mean-field steady-states suggest that, in a spatial version of the model, one can expect the formation of a traveling wave of tumor cells invading the healthy tissue.

\subsection{Spatial mean-field analysis}

\subsubsection{Lattice-Boltzmann equation}

We propose the derivation of a spatio-temporal mean-field approximation of our LGCA model for a spatially distributed system. We define the single cell distribution function as the average value $E$ of the channel occupation numbers:

$$
f_{\sigma, i}=E\left(\eta_{\sigma, i}(\mathbf{r}, k)\right)=\sum_{\boldsymbol{\eta}_{\sigma}} \eta_{\sigma, i}(\mathbf{r}, k) P_{k}\left(\boldsymbol{\eta}_{\sigma}(\mathbf{r}, k)\right),
$$


where $f_{\sigma, i} \in[0,1], i=1, \ldots, \tilde{b}$ and the average is defined over an arbitrary node distribution $P_{k}\left(\boldsymbol{\eta}_{\sigma}(\mathbf{r}, k)\right)$. Moreover, we define the mean node density as

$$
\rho_{\sigma}(\mathbf{r}, k)=E\left(n_{\sigma}(\mathbf{r}, k)\right)=\sum_{i=1}^{\tilde{b}} f_{\sigma, i}(\mathbf{r}, k) .
$$

The term $P_{k}\left(\boldsymbol{\eta}_{\sigma}(\mathbf{r}, k)\right)$ are often complicated due to high correlations among the $\eta_{\sigma, i}$ 's. In order to gain more insight into the automaton dynamics, we apply the mean-field approximation and neglect all on-node (within the channels of one node) and off-node (with neighboring nodes) correlations. Hence, we can write the fully factorized node distribution:

$$
P_{k}\left(\boldsymbol{\eta}_{\sigma}(\mathbf{r}, k)\right)=\prod_{i=1}^{\tilde{b}} f_{\sigma, i}(\mathbf{r}, k)^{\eta_{\sigma, i}(\mathbf{r}, k)}\left(1-f_{\sigma, i}(\mathbf{r}, k)\right)^{1-\eta_{\sigma, i}(\mathbf{r}, k)} .
$$

With some algebra and using this factorization in the micro-dynamical description (2.4), we derive the following mean-field approximation of our LGCA model, which is also called nonlinear lattice Boltzmann equation (LBE):

$$
\begin{aligned}
f_{\sigma, i}\left(\mathbf{r}+m_{\sigma} \mathbf{c}_{i}, k+\tau\right)-f_{\sigma, i}(\mathbf{r}, k) & =\frac{1}{\tilde{b}} \sum_{j=1}^{\tilde{b}} E\left(\eta_{\sigma, j}^{\mathrm{K}}(\mathbf{r}, k)\right)-f_{\sigma, i}(\mathbf{r}, k) \\
& =\sum_{j=1}^{\tilde{b}} \Omega_{i j} f_{\sigma, j}(\mathbf{r}, k)+\tilde{\mathcal{R}}_{\sigma, i}(\mathbf{r}, k) \in[-1,1],
\end{aligned}
$$

where $\Omega=\left(1 / \tilde{b}-\delta_{i j}\right)_{i, j=1, \ldots, \tilde{b}}$ is the transition matrix of the reorientation process. We assume that the mean-field growth terms are independent of the direction of cell movement, meaning that we can write $\tilde{\mathcal{R}}_{\sigma, i}(\mathbf{r}, k)=F_{\sigma}\left(\rho_{r}, \rho_{v}\right) / \tilde{b}$ where the $F_{\sigma}$ 's (for $\sigma \in\{r, v\}$ ) are the mean-field growth laws for a single node as given by (4.7) and (4.8) under the assumption of a well-stirred system.

\subsubsection{Spatio-temporal dynamics}

We derive the macroscopic dynamics of the system by means of the Chapman-Enskog method. We assume a parabolic (diffusive) scaling

$$
\mathbf{x}=\epsilon \mathbf{r} \quad \text { and } \quad t=\epsilon^{2} k
$$

where $(\mathbf{x}, t)$ are continuous variables and $\epsilon$ is the (small) scaling parameter.

The Chapman-Enskog expansion method is based on assuming that a solution of the LBE (4.14), rescaled in time and space as proposed above, can be approximated by $f_{\sigma, i}(\mathbf{x}, t)=\rho_{\sigma}(\mathbf{x}, t) / \tilde{b}$ at the first order, with small corrections at higher orders that are evaluated from perturbation analysis. More precisely, we assume an asymptotic solution of the single cell distribution of the form

$$
f_{\sigma, i}=f_{\sigma, i}^{(0)}+\epsilon f_{\sigma, i}^{(1)}+\epsilon^{2} f_{\sigma, i}^{(2)}+\mathcal{O}\left(\epsilon^{3}\right)
$$


where the $f_{\sigma, i}^{(l)}$, s are the functions of $\mathbf{x}$ and $t$ to be determined.

An important aspect is the scaling of the kinetic term $\tilde{\mathcal{R}}_{\sigma, i}$. We assume that the cell kinetics are scaled according to the macroscopic time scaling, i.e.

$$
\overline{\mathcal{R}}_{\sigma, i} \rightarrow \epsilon^{2} \tilde{\mathcal{R}}_{\sigma, i} .
$$

The above relation implies that the macroscopic growth rate and switching probabilities scale as $r_{m}=\epsilon^{2} \bar{r}_{m} \ll 1, \gamma_{v r}=\epsilon^{2} \bar{\gamma}_{v r} \ll 1$ and $\gamma_{r v}=\epsilon^{2} \bar{\gamma}_{r v} \ll 1$, where $\bar{r}_{m}, \bar{\gamma}_{v r}, \bar{\gamma}_{r v}$ are $\mathcal{O}(1)$ to maintain the key terms of the kinetics in the macroscopic formulation. This means that the development of the approach below is restricted to values of the model parameters that are very small.

The macroscopic quantities of interest are the two cell densities $\rho_{\sigma}$ for $\sigma \in\{r, v\}$. The key to the Chapman-Enskog expansion is to impose that each cell density is completely expressed in terms of $f_{\sigma, i}^{(0)}$. It means that the local mass at a node is entirely accounted for in $\rho_{\sigma}=\sum_{i=1}^{\tilde{b}} f_{\sigma, i}^{(0)}$, which consequently imposes that $\sum_{i=1}^{\tilde{b}} f_{\sigma, i}^{(l)}=0$ for $l \geq 1$.

With some algebra and accounting for resting cells that are, by definition, not allowed to move, the procedure leads to a system of two reaction-diffusion equations

$$
\left\{\begin{aligned}
\frac{\partial \rho_{r}}{\partial t} & =\frac{1}{\tau} \bar{F}_{r}\left(\rho_{r}, \rho_{v}\right) \\
\frac{\partial \rho_{v}}{\partial t} & =\frac{m_{v}^{2}}{\tau \tilde{b}} \nabla^{2} \rho_{v}+\frac{1}{\tau} \bar{F}_{v}\left(\rho_{r}, \rho_{v}\right),
\end{aligned}\right.
$$

where $\bar{F}_{r}$ and $\bar{F}_{v}$ are the macroscopic growth laws already defined in (4.10) and (4.11) however rewritten with $\bar{r}_{m}, \bar{\gamma}_{v r}$ and $\bar{\gamma}_{r v}$ according to the current rescaling. The RDE system (4.18) allows for a macroscopic description of the spatio-temporal behavior of the LGCA model.

Remark that the structure is similar to that proposed in other studies [15, 17]. In particular, in the latter the authors investigate the influence of density-dependent quiescent phases on glioma invasion by considering phenotypic switching mechanisms regulated by the local cell density. Our model recovers the formulation proposed in [17] by assuming that: i) the LGCA mitotic operator (M) depend on the total node density $n(\mathbf{r})=n_{r}(\mathbf{r})+n_{v}(\mathbf{r})$ and ii) the switching probabilities take the form

$$
\begin{aligned}
& \frac{\bar{\gamma}_{r v}}{\beta}=\frac{\Gamma(\rho)}{1-\rho_{v} / b}, \\
& \frac{\bar{\gamma}_{v r}}{b}=\frac{1-\Gamma(\rho)}{1-\rho_{r} / \beta},
\end{aligned}
$$

where $\Gamma(\rho)$ represents the density-dependent switching probability used in [17]. This suggests that our mean-field macroscopic model (4.18) may admit traveling wave solutions. This also suggest that other scaling arguments than those proposed here may yield more complex reaction terms in our RDE system and potentially lead to the formation of complex pattern associated with tumor heterogeneity during invasion as observed in [17]. 


\subsubsection{Comparison of the mean-field approximation and the LGCA dynamics}

In this section, we compare our discrete stochastic (LGCA) and continuous deterministic (meanfield approximation) models with the aim to emphasize the limitations of the macroscopic RDE system (4.18) to capture the particular features that result from the "Go-or-Grow" mechanism.

We solve numerically system (4.18) in one dimension with the open-source numerical simulation package eXtensible Multi-Dimensional Simulator for RDE systems (http://www.xmds.org), which is a code generator that integrates partial differential equations. We use a fourth order Runge-Kutta in the interaction picture (RK4IP). The method is closely related to the split-step Fourier method and is described in detail in [3]. The RK4IP algorithm transforms the problem to an interaction picture to separate the effect of diffusion from the nondiffusive terms, leading to a system of ordinary differential equations with time as the independent variable. The system of equations is solved with a fourth-order Runge-Kutta. The evolution of the system is carried out in both normal space and Fourier space.

We use periodic boundary conditions with a domain sufficiently large to avoid boundary effects and define the initial condition for resting and moving cell densities as symmetric plateau distributions at the center of the domain, matching the initial condition used in the LGCA (i.e. the fully-occupied disc at center of the lattice). We assume radial symmetry of the systems to compare the numerical 1D-continuous and 2D-discrete solutions.

It has already been shown (see [14] for example) that the macroscopic mean-field description generally fails to evaluate the exact behavior of the invasive front as observed in the LGCA model. This is mainly due to the fact that correlations built by the local fluctuating dynamics at the tip of the front are neglected in the macroscopic description (see also the discussion below for critical perspectives). Therefore, we base our comparison on another criterion and focus on the time evolution of the population of each cell type in the tumor colony.

We solve numerically the discrete (LGCA) and continuous (RDE) models with the same parameters $\left(r_{m}, \gamma_{v r}, \gamma_{r v}\right)$ describing the mitotic and switching probabilities, for two cases. The first case corresponds to small values of all three parameters (i.e. accouting for cell kinetics occurring at a much slower timescale than cell movement) as required to apply the Chapman-Enskog method in order to derive the macroscopic RDE system. The second case is based on a particular set of unconstrained parameters. We show the numerical results in Figures 11(a) and (b), respectively.

As expected, the mean-field approximation provides a satisfactory representation of the LGCA dynamics, in terms of cell number in the colony, but only for very low mitotic $\left(r_{m}\right)$ and switching $\left(\gamma_{v r}, \gamma_{r v}\right)$ probabilities satisfying the requirements for the RDE derivation, and fails otherwise. This means that the two major features resulting from the "Go-or-Grow" mechanism (which we found when investigating the LGCA dynamics in Section 3.3.) cannot be captured by our mean-field model since these features are only observed for high values of some of the parameters. This raises the question of the use of macroscopic mean-field approximations to accurately model glioma invasion. 
(a)

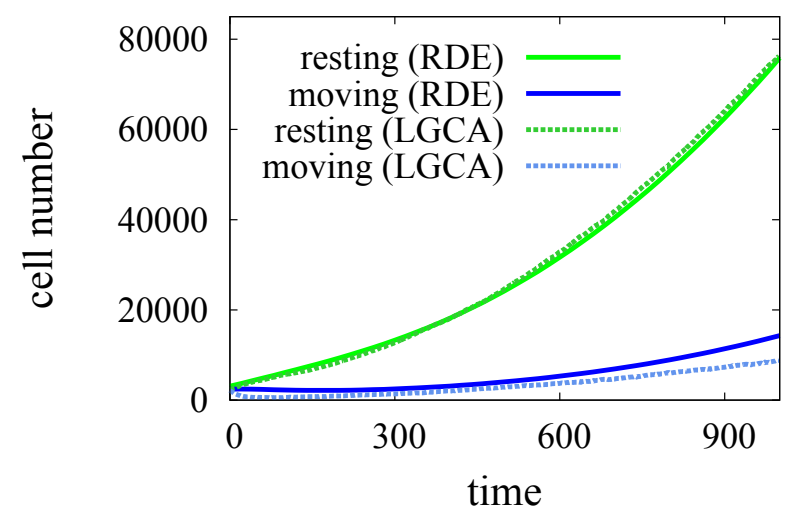

(b)

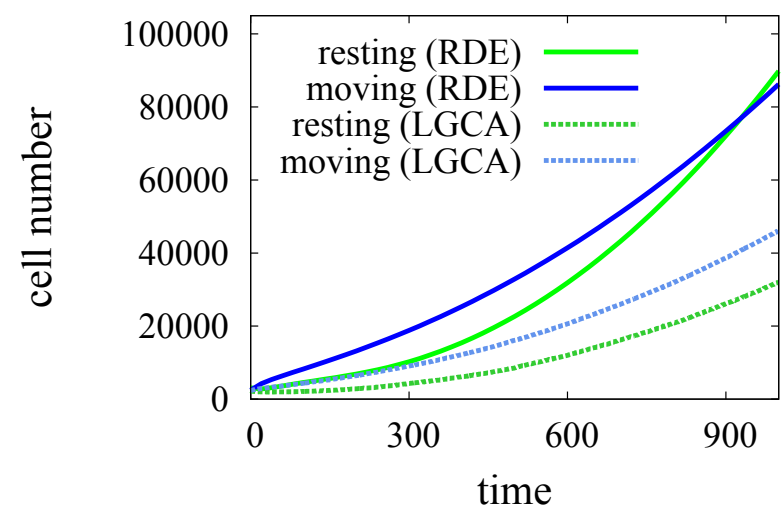

Figure 11: Numerical comparison of the mean-field approximation and the LGCA dynamics. Each figure shows the time evolution of the resting and moving cell numbers calculated from the RDE (solid lines) and LGCA simulations (dashed lines). (a) Good agreement for low mitotic and switching probabilities $\left(r_{m}=0.05, \gamma_{v r}=0.05, \gamma_{r v}=0.01\right)$. (b) The RDE solution fails to match the LGCA dynamics. The assumption on the order of magnitude of the parameters is not satisfied (we use $r_{m}=0.25, \gamma_{v r}=0.01, \gamma_{r v}=0.95$ ) to allow the use of the Chapman-Enskog method, which yields different behaviors of the two models.

\section{Discussion and conclusion}

In this paper, we have analyzed the invasive behavior of avascular glioma tumors under the "Go-orGrow" hypothesis with the help of a lattice-gas cellular automaton model. Our model is based on splitting the tumor cell colony into two sub-populations that are respectively moving and resting, with only the latter being allowed to proliferate.

We have investigated the impact of the "Go-or-Grow" mechanism via the behavior of two relevant observables characterizing invasion dynamics, namely the width of the invasive rim around the tumor bulk and the invasion speed defined as the time derivative of the invasive radius. We have performed a systematic numerical investigation to gain insight into the dependence of the observables on the model parameters, i.e. the mitotic and switching probabilities. We have found that the time-growing invasive zone (observed experimentally in vitro) may be the direct result of the "Go-or-Grow" mechanism. Additionally, we have shown that the invasion speed of a glioma tumor is the non-trivial result of the complex interplay between two interdependent and competing processes that are i) emptying-space, via cell migration, to allow for free space necessary to cell proliferation; ii) filling-space, via proliferation of cells at rest, when free space is available. In particular, the invasive behavior of the whole tumor colony cannot be extrapolated by the sole knowledge of the individual cell phenotype.

Finally, we have derived various mean-field approximations to investigate our LGCA model from a mathematical perspective. Although these approximations are useful since they allow for a simplified description of tumor invasion, we have shown that the main features associated with the "Go-or-Grow" mechanism cannot be captured by such approaches. This raises the issue of 
disregarding the single cell behavior when trying to mathematically understand and predict the invasive dynamics of glioma and suggest that other approximations than those proposed in this paper should be considered.

\subsection{Prediction of traveling front behavior}

An extremely interesting observable of our model is the invasive tumor front, since it has clinical implications that will be discussed below. Although, based on numerical simulations, we have provided a complete analysis of this tumor front in our LGCA model, the quantitative mathematical analysis of such a traveling front, in terms of speed and width, is very challenging in the corresponding macroscopic description of the dynamics and relies on: (i) the stochastic fluctuations of the front (tumor-stroma) interface; (ii) the non-linearities of the macroscopic dynamics description (i.e. the RDE system); (iii) the complexity of the biological phenomena that have to be accounted for.

The front speed can be theoretically estimated by using the macroscopic mean-field description of the system. However, this estimate loses accuracy for large values of the mitotic rate and overestimates the LGCA front speed when one uses a "naive" mean-field approximation [14] as our RDE system (4.18), in which correlations built by the local fluctuating dynamics at the tip of the front are neglected. An alternative mean-field approach has been proposed by Hatzikirou and coworkers, in which a simple RDE model of tumor growth is derived as a cut-off mean-field approximation of a LGCA model [14]. Such a cut-off reaction-diffusion approach enables to phenomenologically include the effect of the foremost single cell fluctuations and would allow, in our current model, for an accurate evaluation of the traveling wave speed.

The second difficulty lies in the non-linear coupling (e.g., the reaction terms (4.10) and (4.11)) of the mean-field RDE system. A potential reduction of the system into a single equation could significantly simplify the analysis of the front dynamics. This would be possible by assuming a timescale separation between the switching and proliferation processes, i.e. by considering phenotypic changes as the result of intracellular processes occurring much faster than cell proliferation. By equipping an appropriate projection method of the fast dynamics on the slow proliferative rates, we could reduce the system to a single reaction-diffusion equation to help estimate the invasion speed of the macroscopic description of our "Go-or-Grow" model.

Finally, another important aspect of tumor invasion is the effect of the microenvironment and its influence on tumor interface dynamics. In our study, we have consciously omitted any microenvironmental factors that could impact the switch between the migratory and proliferative phenotypes, with the aim of deriving a model simple enough to understand the impact of the sole "Go-or-Grow" mechanism on tumor invasion. However, when phenotypic heterogeneity is observed in vitro, phenotypic switches are regulated by either extrinsic or intrinsic factors or by a combination of both [21]. An example of microenvironmental component influencing a change of cell phenotype is the local cell density, which has been shown to have a major impact on the dynamics of glioma invasion [17, 22]. Other microenvironmental cues and, in particular, those associated with the biophysical properties of the healthy tissue surrounding the tumor (e.g., vasculature), are known for being of importance in invasion processes and will be investigated in future studies. 


\subsection{Clinical implications}

A crucial clinical aspect of glioma is the understanding of the invasion dynamics and, in particular, the extent of the infiltration zone of the tumor into the adjacent brain tissue. Indeed, tumor resection, as currently performed, remains quite inefficient since an "invisible" (i.e. with very low density of tumor cells) part of the glioma colony may have invaded the surrounding brain parenchyma and not be detected together with the main tumor bulk. These "invisible" tumor cells are believed to be those responsible for tumor recurrence. An accurate prediction of the infiltration extent would then improve the outcome of tumor resection and thus, of patient's prognosis. However, this region is characterized by a very low density of highly motile cells and can hardly be measured in vivo (at least with current methods). This is the reason why several mathematical models (e.g., $[11,23])$ have been developed to investigate and predict the extent of this region. These models are based on a continuum (mean-field) description of the tumor. As shown in our study, such mean-field descriptions may not capture important features of the invasive front. Therefore, we believe that our modeling approach could be extended and used to account for a more complete picture of glioma invasion, by including microenvironmental features in a 3D tumor model, so as to construct a mathematical estimator of the invasive zone, which does not exist so far.

In our study we have shown that a tumor cell colony regulated by the "Go-or-Grow" mechanism may give rise to an infiltration region whose size increases over time in a nonlinear manner, i.e. characterized by a time-divergent width, as experimentally observed in [20]. Therefore, we argue that this particular coupling of cell proliferation and migration is likely to play a major role in glioma invasion. Despite the theoretical nature of our approach, this feature is a step forward toward a better understanding of the infiltration extent. A naive clinical suggestion would consists in the control (or even the disruption) of the processes regulating the migration/proliferation dichotomy, i.e. the decoupling of the migration and proliferation molecular pathways. Such a direct therapeutic target would indeed allow for a better control of the infiltration zone, but may not be a practical consideration as it may be too disruptive to the healthy tissues. Another more practical option of interest would be to indirectly target the microenvironment, e.g. similar to normalizationlike processes used for vasculature control, to help control environmental conditions that are likely to play an important role in the expression of the tumor cell phenotype.

\section{Appendix}

\subsection{Post-reaction states}

In this Appendix, we present the details of the post-reaction states $\eta_{\sigma, i}^{K}(\mathbf{r}, k)$ defining the cell kinetics. Following equation (2.2), the post-reaction states of the resting and moving tumor cell populations are given by

$$
\begin{aligned}
\eta_{r, i}^{\mathrm{K}}(\mathbf{r}, k) & =\eta_{r, i}(\mathbf{r}, k)+\mathcal{R}_{r, i}(\mathbf{r}, k), \\
\eta_{v, i}^{\mathrm{K}}(\mathbf{r}, k) & =\eta_{v, i}(\mathbf{r}, k)+\mathcal{R}_{v, i}(\mathbf{r}, k) .
\end{aligned}
$$


Following the definition of the cell kinetic rules of the LGCA provided in Section 2.1.2., the terms $\mathcal{R}_{\sigma, i}$ can be written in a stochastic way as

$$
\begin{aligned}
& \mathcal{R}_{r, i}(\mathbf{r}, k)=\xi_{v r, i}\left(1-\eta_{r, i}(\mathbf{r}, k)\right)-\xi_{r v, i} \eta_{r, i}(\mathbf{r}, k)+\xi_{m, i}\left(1-\eta_{r, i}^{S}(\mathbf{r}, k)\right), \\
& \mathcal{R}_{v, i}(\mathbf{r}, k)=\tilde{\xi}_{r v, i}\left(1-\eta_{v, i}(\mathbf{r}, k)\right)-\tilde{\xi}_{v r, i} \eta_{v, i}(\mathbf{r}, k)
\end{aligned}
$$

where

$$
\eta_{r, i}^{S}(\mathbf{r}, k)=\eta_{r, i}(\mathbf{r}, k)+\xi_{v r, i}\left(1-\eta_{r, i}(\mathbf{r}, k)\right)-\xi_{r v, i} \eta_{r, i}(\mathbf{r}, k) .
$$

The variables $\xi$ 's are random Boolean variables used to model either the realization of a phenotypic switching event (i.e. for $\xi_{v r, i}, \xi_{r v, i}, \tilde{\xi}_{r v, i}, \tilde{\xi}_{v r, i}$ ) or a mitotic event (i.e. for $\xi_{m, i}$ ) and satisfy $\sum_{i=1}^{\tilde{b}} \xi_{v r, i}=\sum_{i=1}^{\tilde{b}} \xi_{r v, i}=\sum_{i=1}^{\tilde{b}} \xi_{m, i}=1$ and $\sum_{i=1}^{\tilde{b}} \tilde{\xi}_{r v, i}=\sum_{i=1}^{\tilde{b}} \tilde{\xi}_{v r, i}=1$. The corresponding probabilities are

$$
\begin{aligned}
P\left(\xi_{v r, i}=1\right) & =\gamma_{v r} \frac{n_{v}(\mathbf{r}, k)}{b} \\
P\left(\xi_{r v, i}=1\right) & =\gamma_{r v} \frac{b-n_{v}(\mathbf{r}, k)}{b} \\
P\left(\xi_{m, i}=1\right) & =r_{m} \frac{n_{r}(\mathbf{r}, k)}{\beta}
\end{aligned}
$$

and

$$
\begin{aligned}
& P\left(\tilde{\xi}_{v r, i}=1\right)=\gamma_{v r} \frac{\beta-n_{r}(\mathbf{r}, k)}{\beta} \\
& P\left(\tilde{\xi}_{r v, i}=1\right)=\gamma_{r v} \frac{n_{r}(\mathbf{r}, k)}{\beta}
\end{aligned}
$$

\subsection{Well-stirred system}

In the automaton context, assuming a well-stirred system corresponds to repeatedly apply the transport operator (2.3), which randomly reshuffles the cells on the velocity channels and propagates them to the neighboring nodes, until the system is homogenized and relaxes to a single binomial distribution over the lattice. Phenomenologically, the well-stirred system corresponds to a divergent diffusion coefficient. By applying the mean-field approximation, i.e. neglecting all spatial correlations, we define

$$
P\left(n_{r}=x\right)=\left(\begin{array}{c}
\beta \\
x
\end{array}\right)\left(\frac{\rho_{r}}{\beta}\right)^{x}\left(1-\frac{\rho_{r}}{\beta}\right)^{\beta-x}, \rho_{r}=E\left(n_{r}\right)=\sum_{x=0}^{\beta} x P\left(n_{r}=x\right),
$$

and

$$
P\left(n_{v}=y\right)=\left(\begin{array}{c}
b \\
y
\end{array}\right)\left(\frac{\rho_{v}}{b}\right)^{y}\left(1-\frac{\rho_{v}}{b}\right)^{b-y}, \rho_{v}=E\left(n_{v}\right)=\sum_{y=0}^{b} y P\left(n_{v}=y\right)
$$


where $x \in\{0, \ldots, \beta\}, y \in\{0, \ldots, b\}$ with $\rho_{r}$ and $\rho_{v}$ being the average node densities of the resting and moving populations, respectively.

The transition matrix $\mathbf{W}$ describes the transition from state $\left(n_{r}, n_{v}\right)$ to state $\left(n_{r}^{\prime}, n_{v}^{\prime}\right)$. In order to calculate the transition matrices for the resting and moving cell populations, we derive the transition matrices $\mathbf{W}_{\mathbf{r}}$ and $\mathbf{W}_{\mathbf{v}}$. By looking at $\mathbf{W}$, we identify

$$
\sum_{n_{v}=0}^{b} \sum_{n_{v}^{\prime}=0}^{b} P\left(n_{r}, n_{v}\right) P\left[\left(n_{r}^{\prime}, n_{v}^{\prime}\right) \mid\left(n_{r}, n_{v}\right)\right]=P\left(n_{r}\right) P\left(n_{r}^{\prime} \mid n_{r}\right)
$$

and similarly

$$
\sum_{n_{r}=0}^{\beta} \sum_{n_{r}^{\prime}=0}^{\beta} P\left(n_{r}, n_{v}\right) P\left[\left(n_{r}^{\prime}, n_{v}^{\prime}\right) \mid\left(n_{r}, n_{v}\right)\right]=P\left(n_{v}\right) P\left(n_{v}^{\prime} \mid n_{v}\right) .
$$

Allowing for a well-stirred system, the two populations are perfectly mixed, meaning that we can write $P\left(n_{r}, n_{v}\right)=P\left(n_{r}\right) P\left(n_{v}\right)$. Consequently, we find that

$$
P\left(n_{r}^{\prime} \mid n_{r}\right)=\sum_{n_{v}=0}^{b} P\left(n_{v}\right) \sum_{n_{v}^{\prime}=0}^{b} P\left[\left(n_{r}^{\prime}, n_{v}^{\prime}\right) \mid\left(n_{r}, n_{v}\right)\right],
$$

and

$$
P\left(n_{v}^{\prime} \mid n_{v}\right)=\sum_{n_{r}=0}^{\beta} P\left(n_{r}\right) \sum_{n_{r}^{\prime}=0}^{\beta} P\left[\left(n_{r}^{\prime}, n_{v}^{\prime}\right) \mid\left(n_{r}, n_{v}\right)\right] .
$$

From the transition matrix $\mathbf{W}$, we can thus calculate $\mathbf{W}_{\mathbf{r}}\left(n_{r}, n_{r}^{\prime}\right)=P\left(n_{r}^{\prime} \mid n_{r}\right)$ and $\mathbf{W}_{\mathbf{v}}\left(n_{v}, n_{v}^{\prime}\right)=P\left(n_{v}^{\prime} \mid n_{v}\right)$.

When applying the cell kinetics operator during one time step $\tau$, the node density (for resting and moving cells) may change from $n_{\sigma}$ to $n_{\sigma}+1, \sigma \in\{r, v\}$ with the transition probability

$$
\begin{aligned}
& P^{+}\left(n_{r}\right)=(-1) \cdot \mathbf{W}_{\mathbf{r}}\left(n_{r}, n_{r}-1\right)+1 \cdot \mathbf{W}_{\mathbf{r}}\left(n_{r}, n_{r}+1\right)+2 \cdot \mathbf{W}_{\mathbf{r}}\left(n_{r}, n_{r}+2\right), \\
& P^{+}\left(n_{v}\right)=(-1) \cdot \mathbf{W}_{\mathbf{v}}\left(n_{v}, n_{v}-1\right)+1 \cdot \mathbf{W}_{\mathbf{v}}\left(n_{v}, n_{v}+1\right) .
\end{aligned}
$$

The entire evolution process of $\left\{n_{\sigma}(\cdot, k): k \in \mathbb{N}\right\}$ is a Markov chain. The probabilities $P_{r}^{+}=\sum_{n_{r}=0}^{\beta} P^{+}\left(n_{r}\right) P\left(n_{r}\right)$ and $P_{v}^{+}=\sum_{n_{v}=0}^{b} P^{+}\left(n_{v}\right) P\left(n_{v}\right)$ are the average of the transition probabilities $P^{+}\left(n_{r}\right)$ and $P^{+}\left(n_{v}\right)$ over the binomial distribution on the lattice. The expectation value of the net change in the node density during one time step becomes

$$
\rho_{\sigma}(k+\tau)-\rho_{\sigma}(k)=P_{\sigma}^{+}=F_{\sigma},
$$

which is a forward Kolmogorov equation. The terms $F_{r}$ and $F_{v}$ are the mean-field growth laws for the resting and moving populations, respectively, as provided in (4.7) and (4.8). 


\section{References}

[1] C. Athale, Y. Mansury, T. Deisboeck. Simulating the impact of a molecular 'decision-process' on cellular phenotype and multicellular patterns in brain tumors. J. Theor. Biol., 233 (2005), 469-481.

[2] J.P. Boon, D. Dab, R. Kapral, A. Lawniczak. Lattice gas automata for reactive systems. Phys. Rpts., 273 (1996), 55-147.

[3] B.M. Caradoc-Davies. Vortex Dynamics in Bose-Einstein Condensates. Ph.D. dissertation, University of Otago, Dunedin, New Zealand (2000).

[4] B. Chopard, M. Droz. Cellular Automata Modeling of Physical Systems. Cambridge University Press (1998).

[5] A. Deutsch, S. Dormann. Cellular automaton modeling of biological pattern formation. Birkhäuser (2005).

[6] S. Fedotov, A. Iomin. Migration and Proliferation Dichotomy in Tumor-Cell Invasion. Phys. Rev. Let., 98 (2007), 118101-4.

[7] C.W. Gardiner. Handbook of stochastic methods. Springer, Berlin (1990).

[8] A. Giese, M.A. Loo, N. Tran, D. Haskett, S.W. Coons, M.E. Berens. Dichotomy of astrocytoma migration and proliferation. Int. J. Cancer, 67 (1996), 275-282.

[9] A. Giese, R. Bjerkvig, M.E. Berens, M. Westphal. Cost of Migration: Invasion of Malignant Gliomas and Implications for Treatment. J. Clin. Onc., 21 (8) (2003), 1624-1636.

[10] J. Godlewski, M. Nowicki, A. Bronisz, G. Nuovo, J. Palatini, M. De Lay, J. Van Brocklyn, M. Ostrowski, E. A. Chiocca, S. E. Lawler. MicroRNA-451 regulates LKB1/AMPK signaling and allows adaptation to metabolic stress in glioma cells. Mol. Cell, 37 (2010), 620-32.

[11] H.L.P. Harpold, E.C. Alvord Jr, K.R. Swanson. The evolution of mathematical modeling of glioma proliferation and invasion. J. Neuropathol. Exp. Neurol., 66 (1) (2007), 1-9.

[12] H. Hatzikirou, D. Basanta, M. Simon, C. Schaller, A. Deutsch. 'Go or Grow': the key to the emergence of invasion in tumor progression? Mathematical Medicine and Biology (Published online July 2010), doi:10.1093/imammb/dqq01.

[13] H. Hatzikirou, L. Brusch, A. Deutsch. From cellular automaton rules to an effective macroscopic mean-field description. Acta Phys. Pol. B Proc., 3 (2010), 399-416.

[14] H. Hatzikirou, L. Brusch, C. Schaller, M. Simon, A. Deutsch. Prediction of traveling front behavior in a lattice-gas cellular automaton model for tumor invasion. Comput. Math. Appl., 59 (2010), 2326-2339. 
[15] M.A. Lewis, G. Schmitz. Biological invasion of an organism with separate mobile and stationary states: Modeling and analysis. Forma, 11 (1996), 1-25.

[16] Y. Mansury, M. Diggory, T. Deisboeck. Evolutionary game theory in an agent-based brain tumor model: Exploring the 'Genotype-Phenotype' link. J. Theor. Biol., 238 (2006), 146156.

[17] K. Pham, A. Chauviere, H. Hatzikirou, X. Li, H. M. Byrne, V. Cristini, J. Lowengrub. Density-dependent quiescence in glioma invasion: instability in a simple reaction-diffusion model for the migration/proliferation dichotomy. J. Biol. Dyn. (Published online June 2011), doi:10.1080/17513758.2011.590610.

[18] R.E. Baker, M.J. Simpson. Simulating invasion with cellular automata: connecting cell-scale and population-scale properties. Phys. Rev. E, 76 (2) (2007), 021918.

[19] A.M. Stein, M. O. Nowicki, T. Demuth, M.E. Berens, S.E. Lawler, E.A. Chiocca, L.M. Sander. Estimating the cell density and invasive radius of $3 d$ glioblastoma tumor spheroids grown in vitro. Appl. Optics, 46 (22) (2007), 5110-5118.

[20] A.M. Stein, T. Demuth, D. Mobley, M. Berens, L.K. Sander. A mathematical model of glioblastoma tumor spheroid invasion in a three-dimensional in vitro experiment. Biophys. J., 92 (1) (2007), 356-365.

[21] D. Stockholm, R. Benchaouir, J. Picot, P. Rameau, T.M.A. Neildez, G. Landini, C. LaplaceBuilhe, A. Paldi. The origin of phenotypic heterogeneity in a clonal cell population in vitro. PLoS ONE, 4 (2007), 1-13.

[22] M. Tektonidis, H. Hatzikirou, A. Chauviere, M. Simmon, K. Schaller, A. Deutsch. Identification of intrinsic in vitro cellular mechanisms for glioma invasion. J. Theor. Biol., 287 (2011), 131-147.

[23] C.H. Wang, J.K. Rockhill, M. Mrugala, D.L. Peacock, A. Lai, K. Jusenius, J.M. Wardlaw, T. Cloughesy, A.M. Spence, R. Rockne, et al. Prognostic significance of growth kinetics in newly diagnosed glioblastomas revealed by combining serial imaging with a novel biomathematical model. Cancer Res., 69 (23) (2009), 9133-9140. 